Supporting Information

General remarks on Experiments page S2

Experimental Details of X-ray Structure Determination for

(R)-1,2-Dimethyl-1-(1-naphthyl)-1,2,2-triphenyldisilane ( $R$ )-5 page S3-S5

\title{
Stereoselective Synthesis of Optically Active Disilanes, and Selective Functionalization by the Cleavage of Silicon-Naphthyl Bonds with Bromine
}

\author{
Keigo Suzuki, Yusuke Kawakami*, Devadasan Velmurugan, ${ }^{\dagger}$ Takashi Yamane $^{\ddagger}$ \\ Graduate School of Materials Science, Japan Advanced Institute of Science \& Technology \\ [JAIST], Asahidai 1-1. Tatsunokuchi, Ishikawa 923-1292, Japan \\ †Department of Crystallography \& Biophysics, University of Madras Guindy Campus, \\ Chennai 600025 India \\ ₹ Department of Biotechnology, School of Engineering, Nagoya University, Furo-cho, Chikusa Nagoya \\ 464-8603, Japan \\ *To whom correspondence should be addressed. E-mail : kawakami@ jaist.ac.jp
}




\section{General remarks on Experiments}

${ }^{1} \mathrm{H}(500 \mathrm{MHz}),{ }^{13} \mathrm{C}(125.7 \mathrm{MHz})$, and ${ }^{29} \mathrm{Si}(99.3 \mathrm{MHz})$ NMR spectra were recorded in $\mathrm{CDCl}_{3}$. Chemical shifts are reported in ppm relative to $\mathrm{CHCl}_{3}\left(\delta 7.26\right.$ for $\left.{ }^{1} \mathrm{H}\right), \mathrm{CDCl}_{3}\left(\delta 77.00\right.$ for $\left.{ }^{13} \mathrm{C}\right)$, and tetramethylsilane $(\delta 0.00$ for ${ }^{29} \mathrm{Si}$ ). GC and DI EI-Mass analyses were carried to analyze the molecular weight.. Specific optical rotations were measured with a digital polarimeter. TLC analyses were performed on Merck Kieselgel $60 \mathrm{~F}_{254}$. Column chromatography was carried out using Wakogel C-300. HPLC analysis to determine optical purity was performed with a Daicel Chem. Ind., CHIRALCEL OD [cellulose tris(3,5-dimethylphenylcarbamate] $(0.46 \mathrm{~cm} \phi$ x $25 \mathrm{~cm})$ optically active stationary phase using hexane as an eluent $(0.4 \mathrm{~mL} / \mathrm{min}, 254 \mathrm{~nm})$. Preparative HPLC with CHIRALCEL OD $(2 \mathrm{cm \phi} \times 25 \mathrm{~cm})$ was performed with hexane $(4 \mathrm{~mL} / \mathrm{min}, 254 \mathrm{~nm})$. 


\section{Experimental Details of X-ray Structure Determination for (R)-1,2-Dimethyl-1-(1-naphthyl)-1,2,2-triphenyldisilane $(R)-5$ \\ Data Collection}

A colorless platelet crystal of $\mathrm{C}_{30} \mathrm{H}_{28} \mathrm{Si}_{2}$ having approximate dimensions of $0.30 \times 0.15 \times 0.10 \mathrm{~mm}$ was mounted on a glass fiber. All measurements were made on a Rigaku RAXIS RAPID imaging plate area detector with graphite monochromated $\mathrm{Cu}-\mathrm{K} \alpha$ radiation. Indexing was performed from 3 oscillations that were exposed for 60 seconds. The crystal-to-detector distance was $127.40 \mathrm{~mm}$. Cell constants and an orientation matrix for data collection corresponded to a primitive orthorhombic cell with dimensions:

$$
\begin{aligned}
& \mathrm{a}=10.015(2) \AA \\
& \mathrm{b}=14.070(2) \AA \\
& \mathrm{c}=17.975(3) \AA \\
& \mathrm{V}=2532.8(7) \AA^{3}
\end{aligned}
$$

For $\mathrm{Z}=4$ and F.W. $=444.72$, the calculated density is $1.17 \mathrm{~g} / \mathrm{cm}^{3}$. The systematic absences of:

$$
\begin{array}{ll}
\text { h00: } & h \pm 2 n \\
\text { 0k0: } & k \pm 2 n \\
001: & 1 \pm 2 n
\end{array}
$$

uniquely determine the space group to be:

$$
\mathrm{P} 2{ }_{1}{ }_{1}{ }_{1}(\# 19)
$$

The data were collected at a temperature of $23 \pm 1^{\circ} \mathrm{C}$ to a maximum $2 \theta$ value of $136.4^{\circ}$. A total of 90 oscillation images were collected. A sweep of data was done using $\omega$ scans from 50.0 to $230.0^{\circ}$ in $10.0^{\circ}$ step, at $\chi=50.0^{\circ}$ and $\phi=0.0^{\circ}$. The exposure rate was 30.0 [sec. $/^{\circ}$ ]. A second sweep was performed using $\omega$ scans from 50.0 to $230.0^{\circ}$ in $10.0^{\circ}$ step, at $\chi=50.0^{\circ}$ and $\phi=90.0^{\circ}$. The exposure rate was 30.0 [sec. $/^{\mathrm{O}}$ ] Another sweep was performed using $\omega$ scans from 50.0 to $230.0^{\circ}$ in $10.0^{\circ}$ step, at $\chi=50.0^{\circ}$ and $\phi=195.0^{\circ}$. The exposure rate was 30.0 [sec./ ${ }^{\mathrm{O}}$ ]. Another sweep was performed using $\omega$ scans from 50.0 to $230.0^{\circ}$ in $10.0^{\circ}$ step, at $\chi=50.0^{\circ}$ and $\phi=270.0^{\circ}$. The exposure rate was 30.0 [sec./ ${ }^{\circ}$ ]. Another sweep was performed using $\omega$ scans from 50.0 to $230.0^{\circ}$ in $10.0^{\circ}$ step, at $\chi=0.0^{\circ}$ and $\phi=0.0^{\circ}$. The exposure rate was 30.0 [sec. $/^{\circ}$ ]. The crystal-to-detector distance was $127.40 \mathrm{~mm}$. Readout was performed in the $0.100 \mathrm{~mm}$ pixel mode.

\section{Data Reduction}

Of the 4630 reflections that were collected, 4606 were unique $\left(\mathrm{R}_{\mathrm{int}}=0.000\right)$; equivalent reflections were merged. The linear absorption coefficient, $\mu$, for $\mathrm{Cu}-\mathrm{K} \alpha$ radiation is $13.7 \mathrm{~cm}^{-1}$. An empirical absorption correction based on azimuthal scans of several reflections was applied which resulted in transmission factors ranging from 0.60 to 0.87 . The data were corrected for Lorentz and polarization effects. 


\section{$\underline{\text { Structure Solution and Refinement }}$}

The structure was solved by direct methods ${ }^{1}$ and expanded using Fourier techniques ${ }^{2}$. The non-hydrogen atoms were refined anisotropically. Hydrogen atoms were refined using the riding model. The final cycle of full-matrix least-squares refinement ${ }^{3}$ on $\mathrm{F}$ was based on 3218 observed reflections $(\mathrm{I}>3.00 \sigma(\mathrm{I})$ ) and 318 variable parameters and converged (largest parameter shift was 0.00 times its esd) with unweighted and weighted agreement factors of:

$$
\begin{gathered}
\mathrm{R}=\Sigma\|\mathrm{Fo}|-| \mathrm{Fc}\| / \Sigma|\mathrm{Fo}|=0.034 \\
\mathrm{R}_{\mathrm{W}}=\left[\Sigma \mathrm{w}(|\mathrm{Fo}|-|\mathrm{Fc}|)^{2} / \Sigma \mathrm{w} \mathrm{Fo}^{2}\right]^{1 / 2}=0.037
\end{gathered}
$$

The standard deviation of an observation of unit weight ${ }^{4}$ was 1.04 . A Sheldrick weighting scheme was used. Plots of $\Sigma \mathrm{w}(|\mathrm{Fo}|-|\mathrm{Fc}|)^{2}$ versus $|\mathrm{Fo}|$, reflection order in data collection, $\sin \theta / \lambda$ and various classes of indices showed no unusual trends. The maximum and minimum peaks on the final difference Fourier map corresponded to 0.41 and $-0.21 \mathrm{e}^{-} / \AA^{3}$, respectively. The flack parameter ${ }^{5}$ is $-0.00(3)$ and The friedel pairs is 4582 .

Neutral atom scattering factors were taken from Cromer and Waber6. Anomalous dispersion effects were included in Fcalc ${ }^{7}$; the values for $\Delta \mathrm{f}^{\prime}$ and $\Delta \mathrm{f}^{\prime \prime}$ were those of Creagh and McAuley ${ }^{8}$. The values for the mass attenuation coefficients are those of Creagh and Hubbell ${ }^{9}$. All calculations were performed using the CrystalStructure ${ }^{10,11}$ crystallographic software package.

\section{References}

(1) SIR92: Altomare, A., Cascarano, G., Giacovazzo, C., Guagliardi, A., Burla, M., Polidori, G., and Camalli, M. (1994) J. Appl. Cryst., 27, 435.

(2) DIRDIF99: Beurskens, P.T., Admiraal, G., Beurskens, G., Bosman, W.P., de Gelder, R., Israel, R. and Smits, J.M.M.(1999). The DIRDIF-99 program system, Technical Report of the Crystallography Laboratory, University of Nijmegen, The Netherlands.

(3) Least Squares function minimized:

$$
\Sigma w\left(\left|\mathrm{~F}_{\mathrm{O}}\right|-\left|\mathrm{F}_{\mathrm{C}}\right|\right)^{2} \quad \text { where } \mathrm{w}=\text { Least Squares weights. }
$$

(4) Standard deviation of an observation of unit weight:

$$
\begin{aligned}
& {\left[\Sigma w\left(\left|\mathrm{~F}_{\mathrm{O}}\right|-\left|\mathrm{F}_{\mathrm{C}}\right|\right)^{2} /\left(\mathrm{N}_{\mathrm{O}}-\mathrm{N}_{\mathrm{V}}\right)\right]^{1 / 2}} \\
& \text { where: } \quad \mathrm{N}_{\mathrm{O}}=\text { number of observations } \\
& \qquad \mathrm{N}_{\mathrm{V}}=\text { number of variables }
\end{aligned}
$$

(5) Flack, H. D. (1983), Acta Cryst. A39, 876-881.

(6) Cromer, D. T. \& Waber, J. T.; "International Tables for X-ray Crystallography", Vol. IV, The Kynoch Press, Birmingham, England, Table 2.2 A (1974). 
(7) Ibers, J. A. \& Hamilton, W. C.; Acta Crystallogr., 17, 781 (1964).

(8) Creagh, D. C. \& McAuley, W.J .; "International Tables for Crystallography", Vol C, (A.J.C. Wilson, ed.), Kluwer Academic Publishers, Boston, Table 4.2.6.8, pages 219-222 (1992).

(9) Creagh, D. C. \& Hubbell, J.H..; "International Tables for Crystallography", Vol C, (A.J.C. Wilson, ed.), Kluwer Academic Publishers, Boston, Table 4.2.4.3, pages 200-206 (1992).

(10) CrystalStructure 3.6.0: Crystal Structure Analysis Package, Rigaku and Rigaku/MSC (2000-2004). 9009 New Trails Dr. The Woodlands TX 77381 USA.

(11) CRYSTALS Issue 10: Watkin, D.J., Prout, C.K. Carruthers, J.R. \& Betteridge, P.W. Chemical Crystallography Laboratory, Oxford, UK. (1996)

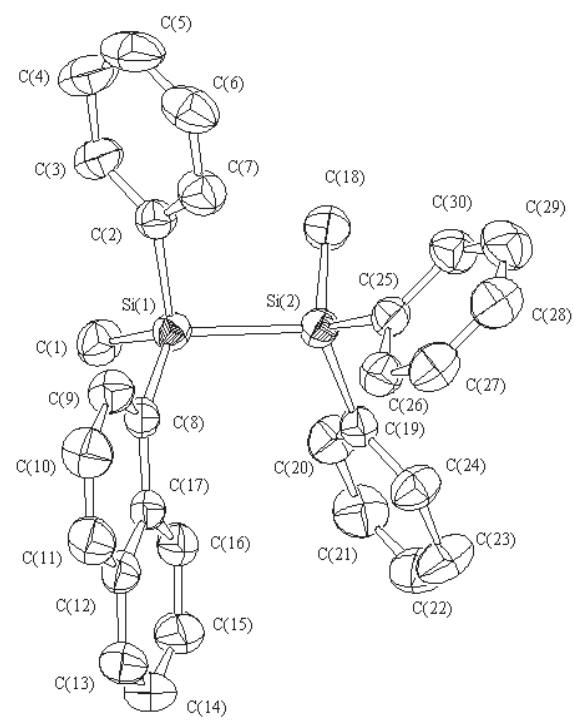

Figure 1. Crystal structure of $(R)-5$. Hydrogen atoms are omitted for clarity. 
EXPERIMENTAL DETAILS

Empirical Formula

Formula Weight

Crystal Color, Habit

Crystal Dimensions

Crystal System

Lattice Type

Indexing Images

Detector Position

Pixel Size

Lattice Parameters

Space Group

$\mathrm{Z}$ value

$\mathrm{D}_{\text {calc }}$

F000

$\mu(\mathrm{CuK} \alpha)$
A. Crystal Data

$\mathrm{C}_{30} \mathrm{H}_{28} \mathrm{Si}_{2}$

444.72

colorless, platelet

$0.30 \times 0.15 \times 0.10 \mathrm{~mm}$

orthorhombic

Primitive

3 oscillations @60.0 seconds

$127.40 \mathrm{~mm}$

$0.100 \mathrm{~mm}$

$a=10.015(2) \AA$

$\mathrm{b}=14.070(2) \AA$

$c=17.975(3) \AA$

$\mathrm{V}=2532.8(7) \AA^{3}$

$\mathrm{P} 2{ }_{1}{ }_{1}{ }_{2}$ (\#19)

4

$1.166 \mathrm{~g} / \mathrm{cm}^{3}$

944.00

$13.67 \mathrm{~cm}^{-1}$ 
B. Intensity Measurements

Diffractometer

Radiation

Detector Aperture

Data Images

$\omega$ oscillation Range $(\chi=50.0, \phi=0.0)$

Exposure Rate

$\omega$ oscillation Range $(\chi=50.0, \phi=90.0)$

Exposure Rate

$\omega$ oscillation Range $(\chi=50.0, \phi=195.0)$

Exposure Rate

$\omega$ oscillation Range $(\chi=50.0, \phi=270.0)$

Exposure Rate

$\omega$ oscillation Range $(\chi=0.0, \phi=0.0)$

Exposure Rate

Detector Position

Pixel Size

$2 \theta_{\max }$

No. of Reflections Measured

Corrections
Rigaku RAXIS-RAPID

$\mathrm{CuK} \alpha(\lambda=1.54187 \AA)$

graphite monochromated

$460 \mathrm{~mm} \times 256 \mathrm{~mm}$

90 exposures

$50.0-230.0^{\mathrm{O}}$

$30.0 \mathrm{sec} . / \mathrm{O}$

$50.0-230.0^{\mathrm{O}}$

$30.0 \mathrm{sec} . \mathrm{I}^{\mathrm{O}}$

$50.0-230.0^{\mathrm{O}}$

$30.0 \mathrm{sec} . / \mathrm{O}$

$50.0-230.0^{\mathrm{O}}$

$30.0 \mathrm{sec} . \mathrm{I}^{\mathrm{O}}$

$50.0-230.0^{\circ}$

$30.0 \mathrm{sec} . /^{\mathrm{O}}$

$127.40 \mathrm{~mm}$

$0.100 \mathrm{~mm}$

$136.4^{\circ}$

Total: 4630

Unique: $4606\left(\mathrm{R}_{\mathrm{int}}=0.000\right)$

Lorentz-polarization

Absorption

(trans. factors: $0.6010-0.8700$ ) 
C. Structure Solution and Refinement

Structure Solution

Refinement

Function Minimized

Least Squares Weights

$2 \theta_{\max }$ cutoff

Anomalous Dispersion

No. Observations (I $>3.00 \sigma(\mathrm{I}))$

No. Variables

Reflection/Parameter Ratio

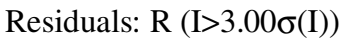

Residuals: Rw (I>3.00\%(I))

Goodness of Fit Indicator

Flack Parameter (Friedel Pairs $=4582)$

Max Shift/Error in Final Cycle

Maximum peak in Final Diff. Map

Minimum peak in Final Diff. Map
Direct Methods (SIR92)

Full-matrix least-squares on $\mathrm{F}$

$\Sigma \mathrm{w}(|\mathrm{Fo}|-|\mathrm{Fc}|)^{2}$

$1 /\left[0.0002 \mathrm{Fo}^{2}+1.0000 \sigma\left(\mathrm{Fo}^{2}\right)\right]$

$136.4^{\circ}$

All non-hydrogen atoms

3218

318

10.12

0.034

0.037

1.041

$-0.00(3)$

0.000

$0.41 \mathrm{e}^{-} / \AA^{3}$

$-0.21 \mathrm{e}^{-/ \AA^{3}}$ 
Table 1. Atomic coordinates and $\mathrm{B}_{\mathrm{iso}} / \mathrm{B}_{\mathrm{eq}}$

\begin{tabular}{|c|c|c|c|c|}
\hline atom & $\mathrm{x}$ & $\mathrm{y}$ & $\mathrm{z}$ & $\mathrm{B}_{\mathrm{eq}}$ \\
\hline $\operatorname{Si}(2)$ & $0.66731(8)$ & $0.13866(6)$ & $0.88310(4)$ & $3.79(2)$ \\
\hline $\operatorname{Si}(1)$ & $0.75266(8)$ & $0.27121(5)$ & $0.81829(4)$ & $3.56(1)$ \\
\hline $\mathrm{C}(17)$ & $0.7945(3)$ & $0.2012(2)$ & $0.6670(1)$ & $3.72(6)$ \\
\hline $\mathrm{C}(19)$ & $0.7553(3)$ & $0.0223(2)$ & $0.8663(1)$ & $3.81(6)$ \\
\hline $\mathrm{C}(8)$ & $0.7319(3)$ & $0.2703(2)$ & $0.7138(1)$ & $3.59(6)$ \\
\hline $\mathrm{C}(25)$ & $0.4868(3)$ & $0.1233(2)$ & $0.8579(2)$ & $3.91(7)$ \\
\hline$C(12)$ & $0.7804(3)$ & $0.2059(2)$ & $0.5882(2)$ & $4.41(7)$ \\
\hline $\mathrm{C}(9)$ & $0.6574(3)$ & $0.3398(2)$ & $0.6799(2)$ & $4.71(7)$ \\
\hline $\mathrm{C}(1)$ & $0.9327(3)$ & $0.2881(2)$ & $0.8432(2)$ & $5.08(8)$ \\
\hline$C(2)$ & $0.6533(3)$ & $0.3731(2)$ & $0.8573(1)$ & $4.09(7)$ \\
\hline$C(16)$ & $0.8759(3)$ & $0.1270(2)$ & $0.6957(2)$ & $4.47(7)$ \\
\hline $\mathrm{C}(18)$ & $0.6857(4)$ & $0.1714(2)$ & $0.9837(2)$ & $5.30(8)$ \\
\hline $\mathrm{C}(11)$ & $0.6993(3)$ & $0.2782(3)$ & $0.5575(2)$ & $5.46(9)$ \\
\hline$C(26)$ & $0.4472(3)$ & $0.1195(2)$ & $0.7831(2)$ & $4.79(8)$ \\
\hline $\mathrm{C}(15)$ & $0.9394(4)$ & $0.0643(3)$ & $0.6499(2)$ & $5.8(1)$ \\
\hline $\mathrm{C}(30)$ & $0.3865(3)$ & $0.1170(2)$ & $0.9106(2)$ & $5.13(8)$ \\
\hline$C(24)$ & $0.6987(3)$ & $-0.0505(2)$ & $0.8259(2)$ & $5.26(8)$ \\
\hline$C(10)$ & $0.6388(4)$ & $0.3430(3)$ & $0.6021(2)$ & $5.66(9)$ \\
\hline $\mathrm{C}(13)$ & $0.8473(4)$ & $0.1397(3)$ & $0.5426(2)$ & $5.79(9)$ \\
\hline $\mathrm{C}(20)$ & $0.8811(3)$ & $0.0053(2)$ & $0.8953(2)$ & $5.38(9)$ \\
\hline $\mathrm{C}(3)$ & $0.7131(4)$ & $0.4448(2)$ & $0.8986(2)$ & $5.29(9)$ \\
\hline $\mathrm{C}(14)$ & $0.9265(4)$ & $0.0719(3)$ & $0.5727(2)$ & $6.5(1)$ \\
\hline $\mathrm{C}(21)$ & $0.9471(4)$ & $-0.0802(3)$ & $0.8840(2)$ & $6.4(1)$ \\
\hline$C(7)$ & $0.5161(4)$ & $0.3788(3)$ & $0.8484(2)$ & $5.43(9)$ \\
\hline $\mathrm{C}(27)$ & $0.3150(4)$ & $0.1069(2)$ & $0.7638(2)$ & $5.65(9)$ \\
\hline $\mathrm{C}(28)$ & $0.2190(3)$ & $0.0987(3)$ & $0.8176(2)$ & $6.1(1)$ \\
\hline $\mathrm{C}(22)$ & $0.8870(4)$ & $-0.1511(3)$ & $0.8446(2)$ & $6.5(1)$ \\
\hline $\mathrm{C}(4)$ & $0.6378(6)$ & $0.5190(3)$ & $0.9285(2)$ & $7.3(1)$ \\
\hline$C(6)$ & $0.4416(4)$ & $0.4537(3)$ & $0.8773(2)$ & $6.6(1)$ \\
\hline $\mathrm{C}(29)$ & $0.2554(4)$ & $0.1037(3)$ & $0.8912(2)$ & $6.5(1)$ \\
\hline $\mathrm{C}(23)$ & $0.7634(4)$ & $-0.1362(2)$ & $0.8149(2)$ & $6.77(9)$ \\
\hline$C(5)$ & $0.5038(5)$ & $0.5233(3)$ & $0.9171(2)$ & 7.4(1) \\
\hline
\end{tabular}

$B_{e q}=8 / 3 \pi^{2}\left(U_{11}\left(a a^{*}\right)^{2}+U_{22}\left(b b^{*}\right)^{2}+U_{33}\left(c c^{*}\right)^{2}+2 U_{12}\left(a a^{*} b b^{*}\right) \cos \gamma+2 U_{13}\left(a a^{*} c c^{*}\right) \cos \beta+2 U_{23}\left(b^{*} c^{*}\right) \cos \alpha\right)$ 
Table 2. Atomic coordinates and B iso involving hydrogens/ $\mathrm{B}_{\mathrm{eq}}$

\begin{tabular}{|c|c|c|c|c|}
\hline atom & $\mathrm{x}$ & $\mathrm{y}$ & $\mathrm{z}$ & $\mathrm{B}_{\mathrm{eq}}$ \\
\hline $\mathrm{H}(1)$ & 0.9853 & 0.2375 & 0.8241 & 6.110 \\
\hline $\mathrm{H}(2)$ & 0.9627 & 0.3465 & 0.8226 & 6.126 \\
\hline $\mathrm{H}(3)$ & 0.9411 & 0.2901 & 0.8958 & 6.118 \\
\hline $\mathrm{H}(4)$ & 0.8071 & 0.4429 & 0.9056 & 6.338 \\
\hline $\mathrm{H}(5)$ & 0.6798 & 0.5666 & 0.9579 & 8.814 \\
\hline $\mathrm{H}(6)$ & 0.4525 & 0.5745 & 0.9363 & 8.942 \\
\hline $\mathrm{H}(7)$ & 0.3481 & 0.4566 & 0.8684 & 7.911 \\
\hline $\mathrm{H}(8)$ & 0.4724 & 0.3286 & 0.8228 & 6.508 \\
\hline $\mathrm{H}(9)$ & 0.6161 & 0.3865 & 0.7102 & 5.638 \\
\hline $\mathrm{H}(10)$ & 0.5852 & 0.3917 & 0.5810 & 6.783 \\
\hline $\mathrm{H}(11)$ & 0.6862 & 0.2817 & 0.5052 & 6.561 \\
\hline $\mathrm{H}(12)$ & 0.8365 & 0.1424 & 0.4901 & 6.936 \\
\hline $\mathrm{H}(13)$ & 0.9725 & 0.0285 & 0.5414 & 7.802 \\
\hline $\mathrm{H}(14)$ & 0.9961 & 0.0174 & 0.6711 & 6.954 \\
\hline $\mathrm{H}(15)$ & 0.8830 & 0.1209 & 0.7482 & 5.351 \\
\hline $\mathrm{H}(16)$ & 0.7778 & 0.1773 & 0.9954 & 6.379 \\
\hline $\mathrm{H}(17)$ & 0.6419 & 0.2301 & 0.9930 & 6.384 \\
\hline $\mathrm{H}(18)$ & 0.6470 & 0.1231 & 1.0137 & 6.367 \\
\hline $\mathrm{H}(19)$ & 0.9235 & 0.0538 & 0.9234 & 6.456 \\
\hline $\mathrm{H}(20)$ & 1.0335 & -0.0898 & 0.9045 & 7.626 \\
\hline $\mathrm{H}(21)$ & 0.9332 & -0.2090 & 0.8360 & 7.750 \\
\hline $\mathrm{H}(22)$ & 0.7199 & -0.1865 & 0.7893 & 8.114 \\
\hline $\mathrm{H}(23)$ & 0.6137 & -0.0405 & 0.8039 & 6.311 \\
\hline $\mathrm{H}(24)$ & 0.5132 & 0.1267 & 0.7456 & 5.733 \\
\hline $\mathrm{H}(25)$ & 0.2906 & 0.1039 & 0.7127 & 6.783 \\
\hline $\mathrm{H}(26)$ & 0.1285 & 0.0887 & 0.8040 & 7.336 \\
\hline $\mathrm{H}(27)$ & 0.1885 & 0.0993 & 0.9285 & 7.814 \\
\hline $\mathrm{H}(28)$ & 0.4106 & 0.1207 & 0.9617 & 6.159 \\
\hline
\end{tabular}


Table 3. Anisotropic Displacement Parameters

\begin{tabular}{|c|c|c|c|c|c|c|}
\hline atom & $\mathrm{U}_{11}$ & $\mathrm{U}_{22}$ & $\mathrm{U}_{33}$ & $\mathrm{U}_{12}$ & $\mathrm{U}_{13}$ & $\mathrm{U}_{23}$ \\
\hline $\operatorname{Si}(2)$ & $0.0502(5)$ & $0.0458(4)$ & $0.0480(4)$ & $-0.0021(4)$ & $-0.0012(4)$ & $-0.0003(4)$ \\
\hline $\operatorname{Si}(1)$ & $0.0437(4)$ & $0.0426(4)$ & $0.0489(4)$ & $0.0016(4)$ & $-0.0014(4)$ & $-0.0010(4)$ \\
\hline$C(17)$ & $0.045(2)$ & $0.048(2)$ & $0.048(2)$ & $-0.009(1)$ & $0.004(1)$ & $-0.001(1)$ \\
\hline $\mathrm{C}(19)$ & $0.048(1)$ & $0.049(2)$ & $0.048(2)$ & $-0.004(2)$ & $0.000(2)$ & $0.004(1)$ \\
\hline $\mathrm{C}(8)$ & $0.045(2)$ & $0.044(1)$ & $0.048(1)$ & $-0.005(1)$ & $0.001(1)$ & $0.002(1)$ \\
\hline$C(25)$ & $0.047(2)$ & $0.043(2)$ & $0.059(2)$ & $0.000(1)$ & $0.003(1)$ & $-0.004(1)$ \\
\hline$C(12)$ & $0.061(2)$ & $0.054(2)$ & $0.053(2)$ & $-0.013(1)$ & $0.003(1)$ & $-0.002(1)$ \\
\hline $\mathrm{C}(9)$ & $0.064(2)$ & $0.057(2)$ & $0.058(2)$ & $0.008(2)$ & $-0.004(2)$ & $0.003(2)$ \\
\hline$C(1)$ & $0.057(2)$ & $0.063(2)$ & $0.074(2)$ & $-0.004(2)$ & $-0.006(2)$ & $-0.007(2)$ \\
\hline $\mathrm{C}(2)$ & $0.061(2)$ & $0.048(2)$ & $0.046(1)$ & $0.005(2)$ & $0.004(1)$ & $0.004(1)$ \\
\hline$C(16)$ & $0.060(2)$ & $0.050(2)$ & $0.060(2)$ & $0.001(2)$ & $0.007(1)$ & $0.002(2)$ \\
\hline $\mathrm{C}(18)$ & $0.078(2)$ & $0.070(2)$ & $0.054(2)$ & $-0.008(2)$ & $-0.001(2)$ & $-0.004(2)$ \\
\hline $\mathrm{C}(11)$ & $0.085(2)$ & $0.079(2)$ & $0.044(2)$ & $-0.006(2)$ & $-0.004(2)$ & $0.006(2)$ \\
\hline$C(26)$ & $0.059(2)$ & $0.062(2)$ & $0.061(2)$ & $-0.005(2)$ & $-0.001(2)$ & $-0.004(2)$ \\
\hline$C(15)$ & $0.077(2)$ & $0.062(2)$ & $0.082(3)$ & $0.016(2)$ & $0.015(2)$ & $-0.005(2)$ \\
\hline $\mathrm{C}(30)$ & $0.052(2)$ & $0.076(2)$ & $0.067(2)$ & $0.002(2)$ & $0.001(2)$ & $-0.003(2)$ \\
\hline $\mathrm{C}(24)$ & $0.059(2)$ & $0.058(2)$ & $0.083(2)$ & $0.006(1)$ & $-0.013(2)$ & $-0.013(2)$ \\
\hline$C(10)$ & $0.080(2)$ & $0.074(2)$ & $0.061(2)$ & $0.011(2)$ & $-0.010(2)$ & $0.015(2)$ \\
\hline $\mathrm{C}(13)$ & $0.088(3)$ & $0.081(2)$ & $0.052(2)$ & $-0.009(2)$ & $0.011(2)$ & $-0.012(2)$ \\
\hline $\mathrm{C}(20)$ & $0.059(2)$ & $0.067(2)$ & $0.079(2)$ & $0.003(2)$ & $-0.012(2)$ & $-0.004(2)$ \\
\hline $\mathrm{C}(3)$ & $0.083(3)$ & $0.058(2)$ & $0.060(2)$ & $0.005(2)$ & $-0.001(2)$ & $-0.010(2)$ \\
\hline$C(14)$ & $0.096(3)$ & $0.074(3)$ & $0.077(3)$ & $0.004(2)$ & $0.022(2)$ & $-0.023(2)$ \\
\hline $\mathrm{C}(21)$ & $0.061(2)$ & $0.089(3)$ & $0.092(3)$ & $0.018(2)$ & $-0.011(2)$ & $-0.002(2)$ \\
\hline$C(7)$ & $0.065(2)$ & $0.067(2)$ & $0.074(2)$ & $0.013(2)$ & $0.007(2)$ & $-0.001(2)$ \\
\hline $\mathrm{C}(27)$ & $0.069(2)$ & $0.069(2)$ & $0.077(2)$ & $0.003(2)$ & $-0.016(2)$ & $-0.006(2)$ \\
\hline $\mathrm{C}(28)$ & $0.043(2)$ & $0.083(2)$ & $0.107(3)$ & $0.004(2)$ & $-0.010(2)$ & $-0.014(2)$ \\
\hline$C(22)$ & $0.087(3)$ & $0.069(2)$ & $0.088(3)$ & $0.030(2)$ & $0.004(2)$ & $-0.007(2)$ \\
\hline$C(4)$ & $0.136(4)$ & $0.070(3)$ & $0.074(2)$ & $0.012(3)$ & $0.001(3)$ & $-0.023(2)$ \\
\hline$C(6)$ & $0.079(3)$ & $0.093(3)$ & $0.078(3)$ & $0.031(2)$ & $0.017(2)$ & $0.008(2)$ \\
\hline $\mathrm{C}(29)$ & $0.052(2)$ & $0.101(3)$ & $0.094(3)$ & $0.004(2)$ & $0.014(2)$ & $-0.009(2)$ \\
\hline $\mathrm{C}(23)$ & $0.092(3)$ & $0.060(2)$ & $0.106(3)$ & $0.014(2)$ & $-0.015(3)$ & $-0.023(2)$ \\
\hline$C(5)$ & $0.126(4)$ & $0.083(3)$ & $0.075(3)$ & $0.046(3)$ & $0.022(3)$ & $-0.007(2)$ \\
\hline
\end{tabular}

The general temperature factor expression: $\exp \left(-2 \pi^{2}\left(a^{*}{ }^{2} U_{11} h^{2}+b^{* 2} U_{22} k^{2}+c^{* 2} U_{33} 1^{2}+2 a * b * U_{12} h k+\right.\right.$ 
$\left.\left.2 \mathrm{a} * \mathrm{c} * \mathrm{U}_{13} \mathrm{hl}+2 \mathrm{~b} * \mathrm{c} * \mathrm{U}_{23} \mathrm{kl}\right)\right)$ 
Table 4. Bond lengths ( $\mathrm{A})$

\begin{tabular}{|c|c|c|c|c|c|}
\hline atom & atom & distance & atom & atom & distance \\
\hline $\operatorname{Si}(2)$ & $\operatorname{Si}(1)$ & $2.359(1)$ & $\operatorname{Si}(2)$ & $\mathrm{C}(19)$ & $1.884(3)$ \\
\hline $\operatorname{Si}(2)$ & $C(25)$ & $1.876(3)$ & $\operatorname{Si}(2)$ & $\mathrm{C}(18)$ & $1.875(3)$ \\
\hline $\operatorname{Si}(1)$ & $\mathrm{C}(8)$ & $1.889(3)$ & $\operatorname{Si}(1)$ & $\mathrm{C}(1)$ & $1.873(3)$ \\
\hline $\operatorname{Si}(1)$ & $\mathrm{C}(2)$ & $1.880(3)$ & $\mathrm{C}(17)$ & $\mathrm{C}(8)$ & $1.430(4)$ \\
\hline $\mathrm{C}(17)$ & $\mathrm{C}(12)$ & $1.426(4)$ & $\mathrm{C}(17)$ & $C(16)$ & $1.421(4)$ \\
\hline$C(19)$ & $C(24)$ & $1.377(4)$ & $\mathrm{C}(19)$ & $\mathrm{C}(20)$ & $1.385(5)$ \\
\hline$C(8)$ & $C(9)$ & $1.373(4)$ & $C(25)$ & $C(26)$ & $1.403(4)$ \\
\hline$C(25)$ & $\mathrm{C}(30)$ & $1.384(4)$ & $\mathrm{C}(12)$ & $\mathrm{C}(11)$ & $1.413(5)$ \\
\hline$C(12)$ & $C(13)$ & $1.410(5)$ & $\mathrm{C}(9)$ & $C(10)$ & $1.411(5)$ \\
\hline$C(2)$ & $\mathrm{C}(3)$ & $1.389(4)$ & $\mathrm{C}(2)$ & $C(7)$ & $1.385(5)$ \\
\hline$C(16)$ & $C(15)$ & $1.364(5)$ & $\mathrm{C}(11)$ & $\mathrm{C}(10)$ & $1.357(5)$ \\
\hline$C(26)$ & $\mathrm{C}(27)$ & $1.380(5)$ & $\mathrm{C}(15)$ & $\mathrm{C}(14)$ & $1.397(6)$ \\
\hline$C(30)$ & $C(29)$ & $1.372(5)$ & $\mathrm{C}(24)$ & $\mathrm{C}(23)$ & $1.383(5)$ \\
\hline $\mathrm{C}(13)$ & $\mathrm{C}(14)$ & $1.353(6)$ & $\mathrm{C}(20)$ & $\mathrm{C}(21)$ & $1.388(5)$ \\
\hline $\mathrm{C}(3)$ & $\mathrm{C}(4)$ & $1.396(5)$ & $\mathrm{C}(21)$ & $\mathrm{C}(22)$ & $1.363(6)$ \\
\hline$C(7)$ & $C(6)$ & $1.391(5)$ & $\mathrm{C}(27)$ & $\mathrm{C}(28)$ & $1.369(5)$ \\
\hline $\mathrm{C}(28)$ & C(29) & $1.373(6)$ & $\mathrm{C}(22)$ & $\mathrm{C}(23)$ & $1.364(6)$ \\
\hline
\end{tabular}


C(4)

C(5)

$1.359(7)$

C(6)

C(5)

1.364(6)

- S14- 
Table 5. Bond lengths involving hydrogens ( $($ )

$\begin{array}{llllll}\text { atom } & \text { atom } & \text { distance } & \text { atom } & \text { atom } & \text { distance } \\ \mathrm{C}(9) & \mathrm{H}(9) & 0.9500 & \mathrm{C}(1) & \mathrm{H}(1) & 0.9500 \\ \mathrm{C}(1) & \mathrm{H}(2) & 0.9500 & \mathrm{C}(1) & \mathrm{H}(3) & 0.9500 \\ \mathrm{C}(16) & \mathrm{H}(15) & 0.9500 & \mathrm{C}(18) & \mathrm{H}(16) & 0.9500 \\ \mathrm{C}(18) & \mathrm{H}(17) & 0.9500 & \mathrm{C}(18) & \mathrm{H}(18) & 0.9500 \\ \mathrm{C}(11) & \mathrm{H}(11) & 0.9499 & \mathrm{C}(26) & \mathrm{H}(24) & 0.9500 \\ \mathrm{C}(15) & \mathrm{H}(14) & 0.9500 & \mathrm{C}(30) & \mathrm{H}(28) & 0.9500 \\ \mathrm{C}(24) & \mathrm{H}(23) & 0.9500 & \mathrm{C}(10) & \mathrm{H}(10) & 0.9500 \\ \mathrm{C}(13) & \mathrm{H}(12) & 0.9500 & \mathrm{C}(20) & \mathrm{H}(19) & 0.9500 \\ \mathrm{C}(3) & \mathrm{H}(4) & 0.9500 & \mathrm{C}(14) & \mathrm{H}(13) & 0.9500 \\ \mathrm{C}(21) & \mathrm{H}(20) & 0.9500 & \mathrm{C}(7) & \mathrm{H}(8) & 0.9499 \\ \mathrm{C}(27) & \mathrm{H}(25) & 0.9501 & \mathrm{C}(28) & \mathrm{H}(26) & 0.9500 \\ \mathrm{C}(22) & \mathrm{H}(21) & 0.9501 & \mathrm{C}(4) & \mathrm{H}(5) & 0.9500 \\ \mathrm{C}(6) & \mathrm{H}(7) & 0.9500 & \mathrm{C}(29) & \mathrm{H}(27) & 0.9500 \\ \mathrm{C}(23) & \mathrm{H}(22) & 0.9500 & \mathrm{C}(5) & \mathrm{H}(6) & 0.9500\end{array}$


Table 6. Bond angles $\left({ }^{\circ}\right)$

\begin{tabular}{|c|c|c|c|c|c|c|c|}
\hline atom & atom & atom & angle & atom & atom & atom & angle \\
\hline$C(19)$ & $\mathrm{Si}(2)$ & $\mathrm{Si}(1)$ & 116.01(9) & $C(25)$ & $\mathrm{Si}(2)$ & $\operatorname{Si}(1)$ & $108.7(1)$ \\
\hline $\mathrm{Si}(2)$ & $\operatorname{Si}(1)$ & $\mathrm{C}(1)$ & $109.3(1)$ & $\mathrm{Si}(2)$ & $\operatorname{Si}(1)$ & $\mathrm{C}(2)$ & $103.11(9)$ \\
\hline $\mathrm{C}(18)$ & $\mathrm{Si}(2)$ & $\operatorname{Si}(1)$ & $104.3(1)$ & $\mathrm{Si}(2)$ & $\operatorname{Si}(1)$ & $\mathrm{C}(8)$ & $116.44(9)$ \\
\hline$C(25)$ & $\mathrm{Si}(2)$ & $\mathrm{C}(19)$ & 108.2(1) & $\mathrm{C}(18)$ & $\mathrm{Si}(2)$ & $C(19)$ & $108.8(1)$ \\
\hline $\operatorname{Si}(2)$ & $\mathrm{C}(19)$ & $\mathrm{C}(24)$ & $122.6(2)$ & $\operatorname{Si}(2)$ & $C(19)$ & $C(20)$ & $121.0(2)$ \\
\hline$C(18)$ & $\mathrm{Si}(2)$ & $\mathrm{C}(25)$ & $110.8(1)$ & $\operatorname{Si}(2)$ & $C(25)$ & $C(26)$ & $120.5(2)$ \\
\hline $\operatorname{Si}(2)$ & $C(25)$ & $\mathrm{C}(30)$ & $122.8(2)$ & $\mathrm{C}(1)$ & $\operatorname{Si}(1)$ & $\mathrm{C}(8)$ & 110.1(1) \\
\hline$C(2)$ & $\mathrm{Si}(1)$ & $\mathrm{C}(8)$ & $108.5(1)$ & $\operatorname{Si}(1)$ & $\mathrm{C}(8)$ & $C(17)$ & $122.8(2)$ \\
\hline $\operatorname{Si}(1)$ & $\mathrm{C}(8)$ & $\mathrm{C}(9)$ & $119.8(2)$ & $C(2)$ & $\operatorname{Si}(1)$ & $\mathrm{C}(1)$ & $108.9(1)$ \\
\hline $\operatorname{Si}(1)$ & $\mathrm{C}(2)$ & $C(3)$ & $121.6(2)$ & $\operatorname{Si}(1)$ & $\mathrm{C}(2)$ & $C(7)$ & $121.8(2)$ \\
\hline$C(17)$ & $\mathrm{C}(8)$ & $\mathrm{C}(9)$ & $117.4(2)$ & $C(16)$ & $C(17)$ & $\mathrm{C}(8)$ & $122.5(2)$ \\
\hline$C(12)$ & $\mathrm{C}(17)$ & $\mathrm{C}(8)$ & $120.6(2)$ & $C(16)$ & $C(17)$ & $C(12)$ & $116.9(3)$ \\
\hline$C(17)$ & $\mathrm{C}(12)$ & $\mathrm{C}(11)$ & $118.6(3)$ & $C(17)$ & $C(12)$ & $C(13)$ & $119.9(3)$ \\
\hline$C(17)$ & $C(16)$ & $C(15)$ & $121.6(3)$ & $C(20)$ & $\mathrm{C}(19)$ & $\mathrm{C}(24)$ & $116.4(3)$ \\
\hline $\mathrm{C}(19)$ & $\mathrm{C}(24)$ & $\mathrm{C}(23)$ & 122.1(3) & $\mathrm{C}(19)$ & $C(20)$ & $\mathrm{C}(21)$ & 121.8(3) \\
\hline $\mathrm{C}(8)$ & $\mathrm{C}(9)$ & $\mathrm{C}(10)$ & $122.4(3)$ & $\mathrm{C}(30)$ & $C(25)$ & $C(26)$ & $116.7(3)$ \\
\hline$C(25)$ & $C(26)$ & $\mathrm{C}(27)$ & $121.2(3)$ & $C(25)$ & $C(30)$ & $C(29)$ & $121.9(3)$ \\
\hline $\mathrm{C}(12)$ & $\mathrm{C}(11)$ & $\mathrm{C}(10)$ & $120.6(3)$ & $\mathrm{C}(13)$ & $C(12)$ & $\mathrm{C}(11)$ & $121.5(3)$ \\
\hline $\mathrm{C}(12)$ & $\mathrm{C}(13)$ & $\mathrm{C}(14)$ & $120.8(3)$ & $\mathrm{C}(9)$ & $C(10)$ & $\mathrm{C}(11)$ & $120.4(3)$ \\
\hline$C(7)$ & $\mathrm{C}(2)$ & $C(3)$ & $116.6(3)$ & $C(2)$ & $\mathrm{C}(3)$ & $\mathrm{C}(4)$ & 121.1(3) \\
\hline $\mathrm{C}(2)$ & $\mathrm{C}(7)$ & $C(6)$ & $122.2(3)$ & $C(16)$ & $C(15)$ & $C(14)$ & $120.5(3)$ \\
\hline$C(26)$ & $\mathrm{C}(27)$ & $\mathrm{C}(28)$ & $120.4(3)$ & $\mathrm{C}(15)$ & $C(14)$ & $\mathrm{C}(13)$ & $120.3(4)$ \\
\hline$C(30)$ & $\mathrm{C}(29)$ & $\mathrm{C}(28)$ & $120.4(3)$ & $C(24)$ & $C(23)$ & $\mathrm{C}(22)$ & $120.2(3)$ \\
\hline$C(20)$ & $\mathrm{C}(21)$ & $\mathrm{C}(22)$ & $120.0(3)$ & $C(3)$ & $\mathrm{C}(4)$ & $C(5)$ & $120.6(4)$ \\
\hline $\mathrm{C}(21)$ & $\mathrm{C}(22)$ & $\mathrm{C}(23)$ & $119.5(4)$ & $C(7)$ & $\mathrm{C}(6)$ & $\mathrm{C}(5)$ & 119.7(4) \\
\hline $\mathrm{C}(27)$ & $\mathrm{C}(28)$ & $\mathrm{C}(29)$ & $119.4(3)$ & $\mathrm{C}(4)$ & $\mathrm{C}(5)$ & $C(6)$ & $119.9(4)$ \\
\hline
\end{tabular}


Table 7. Bond angles involving hydrogens $\left({ }^{\circ}\right)$

\begin{tabular}{|c|c|c|c|c|c|c|c|}
\hline atom & atom & atom & angle & atom & atom & atom & angle \\
\hline $\operatorname{Si}(2)$ & $\mathrm{C}(18)$ & $\mathrm{H}(16)$ & 109.3 & $\operatorname{Si}(2)$ & $\mathrm{C}(18)$ & $\mathrm{H}(17)$ & 109.8 \\
\hline $\operatorname{Si}(2)$ & $\mathrm{C}(18)$ & $\mathrm{H}(18)$ & 109.4 & $\operatorname{Si}(1)$ & $\mathrm{C}(1)$ & $\mathrm{H}(3)$ & 109.1 \\
\hline $\operatorname{Si}(1)$ & $\mathrm{C}(1)$ & $\mathrm{H}(1)$ & 110.6 & $\operatorname{Si}(1)$ & $\mathrm{C}(1)$ & $\mathrm{H}(2)$ & 108.7 \\
\hline $\mathrm{C}(17)$ & $C(16)$ & $\mathrm{H}(15)$ & 118.0 & $\mathrm{C}(19)$ & $\mathrm{C}(24)$ & $\mathrm{H}(23)$ & 118.6 \\
\hline C(19) & $\mathrm{C}(20)$ & $\mathrm{H}(19)$ & 118.9 & $\mathrm{C}(8)$ & $\mathrm{C}(9)$ & $\mathrm{H}(9)$ & 118.3 \\
\hline$C(25)$ & $C(26)$ & $\mathrm{H}(24)$ & 118.7 & $\mathrm{C}(25)$ & $\mathrm{C}(30)$ & $\mathrm{H}(28)$ & 118.3 \\
\hline$C(12)$ & $\mathrm{C}(11)$ & $\mathrm{H}(11)$ & 120.2 & $\mathrm{C}(12)$ & $\mathrm{C}(13)$ & $\mathrm{H}(12)$ & 119.7 \\
\hline $\mathrm{H}(9)$ & $\mathrm{C}(9)$ & $\mathrm{C}(10)$ & 119.3 & $\mathrm{C}(9)$ & $C(10)$ & $\mathrm{H}(10)$ & 119.6 \\
\hline $\mathrm{H}(3)$ & $\mathrm{C}(1)$ & $\mathrm{H}(1)$ & 109.5 & $\mathrm{H}(2)$ & $\mathrm{C}(1)$ & $\mathrm{H}(1)$ & 109.5 \\
\hline $\mathrm{H}(3)$ & $\mathrm{C}(1)$ & $\mathrm{H}(2)$ & 109.5 & $\mathrm{C}(2)$ & $\mathrm{C}(3)$ & $\mathrm{H}(4)$ & 118.6 \\
\hline$C(2)$ & $C(7)$ & $\mathrm{H}(8)$ & 118.0 & $\mathrm{H}(15)$ & $C(16)$ & $C(15)$ & 120.4 \\
\hline$C(16)$ & $C(15)$ & $\mathrm{H}(14)$ & 119.1 & $\mathrm{H}(17)$ & $\mathrm{C}(18)$ & $\mathrm{H}(16)$ & 109.5 \\
\hline $\mathrm{H}(18)$ & $\mathrm{C}(18)$ & $\mathrm{H}(16)$ & 109.5 & $\mathrm{H}(18)$ & $C(18)$ & $\mathrm{H}(17)$ & 109.5 \\
\hline $\mathrm{H}(11)$ & $\mathrm{C}(11)$ & $\mathrm{C}(10)$ & 119.2 & $\mathrm{C}(11)$ & $C(10)$ & $\mathrm{H}(10)$ & 120.1 \\
\hline $\mathrm{H}(24)$ & $C(26)$ & $\mathrm{C}(27)$ & 120.1 & $C(26)$ & $\mathrm{C}(27)$ & $\mathrm{H}(25)$ & 119.7 \\
\hline $\mathrm{H}(14)$ & $\mathrm{C}(15)$ & $\mathrm{C}(14)$ & 120.4 & $C(15)$ & $\mathrm{C}(14)$ & $\mathrm{H}(13)$ & 119.6 \\
\hline $\mathrm{H}(28)$ & $\mathrm{C}(30)$ & $\mathrm{C}(29)$ & 119.8 & $\mathrm{C}(30)$ & C(29) & $\mathrm{H}(27)$ & 120.3 \\
\hline $\mathrm{H}(23)$ & $\mathrm{C}(24)$ & $\mathrm{C}(23)$ & 119.3 & $\mathrm{C}(24)$ & $\mathrm{C}(23)$ & $\mathrm{H}(22)$ & 120.2 \\
\hline $\mathrm{H}(12)$ & $\mathrm{C}(13)$ & $\mathrm{C}(14)$ & 119.5 & $\mathrm{C}(13)$ & $\mathrm{C}(14)$ & $\mathrm{H}(13)$ & 120.0 \\
\hline $\mathrm{H}(19)$ & $C(20)$ & $\mathrm{C}(21)$ & 119.3 & $\mathrm{C}(20)$ & $\mathrm{C}(21)$ & $\mathrm{H}(20)$ & 119.9 \\
\hline $\mathrm{H}(4)$ & $\mathrm{C}(3)$ & $\mathrm{C}(4)$ & 120.3 & $\mathrm{C}(3)$ & $\mathrm{C}(4)$ & $\mathrm{H}(5)$ & 120.1 \\
\hline $\mathrm{H}(20)$ & $\mathrm{C}(21)$ & $\mathrm{C}(22)$ & 120.0 & $\mathrm{C}(21)$ & $\mathrm{C}(22)$ & $\mathrm{H}(21)$ & 119.8 \\
\hline $\mathrm{H}(8)$ & $C(7)$ & $C(6)$ & 119.8 & $\mathrm{C}(7)$ & $\mathrm{C}(6)$ & $\mathrm{H}(7)$ & 119.9 \\
\hline $\mathrm{H}(25)$ & $\mathrm{C}(27)$ & $\mathrm{C}(28)$ & 119.9 & $\mathrm{C}(27)$ & $\mathrm{C}(28)$ & $\mathrm{H}(26)$ & 120.0 \\
\hline $\mathrm{C}(28)$ & $\mathrm{C}(29)$ & $\mathrm{H}(27)$ & 119.3 & $\mathrm{H}(26)$ & $\mathrm{C}(28)$ & $\mathrm{C}(29)$ & 120.6 \\
\hline $\mathrm{C}(22)$ & $\mathrm{C}(23)$ & $\mathrm{H}(22)$ & 119.5 & $\mathrm{H}(21)$ & $\mathrm{C}(22)$ & $C(23)$ & 120.6 \\
\hline $\mathrm{H}(5)$ & $\mathrm{C}(4)$ & $\mathrm{C}(5)$ & 119.3 & $\mathrm{C}(4)$ & $\mathrm{C}(5)$ & $\mathrm{H}(6)$ & 120.9 \\
\hline $\mathrm{H}(7)$ & $\mathrm{C}(6)$ & $\mathrm{C}(5)$ & 120.4 & $\mathrm{C}(6)$ & $\mathrm{C}(5)$ & $\mathrm{H}(6)$ & 119.3 \\
\hline
\end{tabular}


Table 8. Torsion Angles $\left({ }^{\mathrm{O}}\right)$

\begin{tabular}{|c|c|c|c|c|c|c|c|c|c|}
\hline atom1 & atom2 & atom3 & atom4 & angle & atom1 & atom2 & atom3 & atom4 & angle \\
\hline $\mathrm{C}(19)$ & $\operatorname{Si}(2)$ & $\mathrm{Si}(1)$ & $\mathrm{C}(8)$ & $67.4(1)$ & $\mathrm{C}(19)$ & $\operatorname{Si}(2)$ & $\mathrm{Si}(1)$ & $\mathrm{C}(1)$ & $-58.2(1)$ \\
\hline$C(19)$ & $\operatorname{Si}(2)$ & $\mathrm{Si}(1)$ & $\mathrm{C}(2)$ & $-173.9(1)$ & $C(25)$ & $\operatorname{Si}(2)$ & $\mathrm{Si}(1)$ & $\mathrm{C}(8)$ & $-54.7(1)$ \\
\hline$C(25)$ & $\operatorname{Si}(2)$ & $\mathrm{Si}(1)$ & $\mathrm{C}(1)$ & 179.7(1) & $C(25)$ & $\operatorname{Si}(2)$ & $\operatorname{Si}(1)$ & $\mathrm{C}(2)$ & $64.0(1)$ \\
\hline $\mathrm{C}(18)$ & $\operatorname{Si}(2)$ & $\mathrm{Si}(1)$ & $\mathrm{C}(8)$ & $-173.0(1)$ & $\mathrm{C}(18)$ & $\operatorname{Si}(2)$ & $\mathrm{Si}(1)$ & $\mathrm{C}(1)$ & $61.4(1)$ \\
\hline $\mathrm{C}(18)$ & $\operatorname{Si}(2)$ & $\mathrm{Si}(1)$ & $\mathrm{C}(2)$ & $-54.3(1)$ & $\operatorname{Si}(1)$ & $\operatorname{Si}(2)$ & $\mathrm{C}(19)$ & $\mathrm{C}(24)$ & $-108.5(2)$ \\
\hline $\operatorname{Si}(1)$ & $\operatorname{Si}(2)$ & $\mathrm{C}(19)$ & $\mathrm{C}(20)$ & $72.4(3)$ & $C(25)$ & $\mathrm{Si}(2)$ & $\mathrm{C}(19)$ & $\mathrm{C}(24)$ & $13.9(3)$ \\
\hline$C(25)$ & $\operatorname{Si}(2)$ & $C(19)$ & $\mathrm{C}(20)$ & $-165.2(2)$ & $\mathrm{C}(18)$ & $\operatorname{Si}(2)$ & $C(19)$ & $\mathrm{C}(24)$ & $134.3(2)$ \\
\hline $\mathrm{C}(18)$ & $\operatorname{Si}(2)$ & $\mathrm{C}(19)$ & $\mathrm{C}(20)$ & $-44.7(3)$ & $\operatorname{Si}(1)$ & $\operatorname{Si}(2)$ & $C(25)$ & $\mathrm{C}(26)$ & $51.9(3)$ \\
\hline $\operatorname{Si}(1)$ & $\operatorname{Si}(2)$ & $C(25)$ & $\mathrm{C}(30)$ & $-126.9(2)$ & $\mathrm{C}(19)$ & $\operatorname{Si}(2)$ & $C(25)$ & $\mathrm{C}(26)$ & $-74.9(2)$ \\
\hline $\mathrm{C}(19)$ & $\operatorname{Si}(2)$ & $C(25)$ & $\mathrm{C}(30)$ & $106.3(2)$ & $\mathrm{C}(18)$ & $\mathrm{Si}(2)$ & $C(25)$ & $C(26)$ & $165.9(2)$ \\
\hline $\mathrm{C}(18)$ & $\operatorname{Si}(2)$ & $C(25)$ & $\mathrm{C}(30)$ & $-12.8(3)$ & $\operatorname{Si}(2)$ & $\operatorname{Si}(1)$ & $\mathrm{C}(8)$ & $\mathrm{C}(17)$ & $-64.1(3)$ \\
\hline $\operatorname{Si}(2)$ & $\operatorname{Si}(1)$ & $\mathrm{C}(8)$ & $\mathrm{C}(9)$ & $118.0(2)$ & $\mathrm{C}(1)$ & $\operatorname{Si}(1)$ & $\mathrm{C}(8)$ & $\mathrm{C}(17)$ & $61.1(2)$ \\
\hline $\mathrm{C}(1)$ & $\mathrm{Si}(1)$ & $\mathrm{C}(8)$ & $C(9)$ & $-116.8(2)$ & $\mathrm{C}(2)$ & $\operatorname{Si}(1)$ & $\mathrm{C}(8)$ & $\mathrm{C}(17)$ & $-179.8(2)$ \\
\hline $\mathrm{C}(2)$ & $\operatorname{Si}(1)$ & $\mathrm{C}(8)$ & $\mathrm{C}(9)$ & $2.3(3)$ & $\mathrm{Si}(2)$ & $\operatorname{Si}(1)$ & $\mathrm{C}(2)$ & $\mathrm{C}(3)$ & $114.8(2)$ \\
\hline $\mathrm{Si}(2)$ & $\operatorname{Si}(1)$ & $\mathrm{C}(2)$ & $\mathrm{C}(7)$ & $-63.0(2)$ & $\mathrm{C}(8)$ & $\mathrm{Si}(1)$ & $\mathrm{C}(2)$ & $\mathrm{C}(3)$ & $-121.2(2)$ \\
\hline $\mathrm{C}(8)$ & $\operatorname{Si}(1)$ & $\mathrm{C}(2)$ & $C(7)$ & $61.0(3)$ & $\mathrm{C}(1)$ & $\operatorname{Si}(1)$ & $\mathrm{C}(2)$ & $\mathrm{C}(3)$ & $-1.3(3)$ \\
\hline $\mathrm{C}(1)$ & $\operatorname{Si}(1)$ & $\mathrm{C}(2)$ & $\mathrm{C}(7)$ & $-179.1(2)$ & $\mathrm{C}(12)$ & $\mathrm{C}(17)$ & $\mathrm{C}(8)$ & $\operatorname{Si}(1)$ & $-177.3(2)$ \\
\hline$C(12)$ & $\mathrm{C}(17)$ & $\mathrm{C}(8)$ & $C(9)$ & $0.7(4)$ & $C(16)$ & $\mathrm{C}(17)$ & $\mathrm{C}(8)$ & $\operatorname{Si}(1)$ & $0.9(4)$ \\
\hline$C(16)$ & $\mathrm{C}(17)$ & $\mathrm{C}(8)$ & $\mathrm{C}(9)$ & $178.9(3)$ & $\mathrm{C}(8)$ & $\mathrm{C}(17)$ & $\mathrm{C}(12)$ & $\mathrm{C}(11)$ & $-2.3(4)$ \\
\hline $\mathrm{C}(8)$ & $C(17)$ & $C(12)$ & $\mathrm{C}(13)$ & $177.4(3)$ & $C(16)$ & $\mathrm{C}(17)$ & $C(12)$ & $\mathrm{C}(11)$ & $179.4(3)$ \\
\hline$C(16)$ & $\mathrm{C}(17)$ & $\mathrm{C}(12)$ & $\mathrm{C}(13)$ & $-0.9(4)$ & $\mathrm{C}(8)$ & $\mathrm{C}(17)$ & $C(16)$ & $\mathrm{C}(15)$ & $-177.1(3)$ \\
\hline $\mathrm{C}(12)$ & $\mathrm{C}(17)$ & $C(16)$ & $\mathrm{C}(15)$ & $1.1(4)$ & $\mathrm{Si}(2)$ & $\mathrm{C}(19)$ & $C(24)$ & $\mathrm{C}(23)$ & $-179.0(3)$ \\
\hline $\mathrm{C}(20)$ & $\mathrm{C}(19)$ & $C(24)$ & $\mathrm{C}(23)$ & $0.1(4)$ & $\operatorname{Si}(2)$ & $\mathrm{C}(19)$ & $\mathrm{C}(20)$ & $\mathrm{C}(21)$ & $179.5(3)$ \\
\hline $\mathrm{C}(24)$ & $\mathrm{C}(19)$ & $C(20)$ & $\mathrm{C}(21)$ & $0.4(5)$ & $\operatorname{Si}(1)$ & $\mathrm{C}(8)$ & $\mathrm{C}(9)$ & $\mathrm{C}(10)$ & 179.7(3) \\
\hline $\mathrm{C}(17)$ & $\mathrm{C}(8)$ & $\mathrm{C}(9)$ & $\mathrm{C}(10)$ & $1.7(4)$ & $\mathrm{Si}(2)$ & $\mathrm{C}(25)$ & $C(26)$ & $\mathrm{C}(27)$ & 179.1(3) \\
\hline $\mathrm{C}(30)$ & $C(25)$ & $C(26)$ & $\mathrm{C}(27)$ & $-2.0(4)$ & $\mathrm{Si}(2)$ & $\mathrm{C}(25)$ & $C(30)$ & $\mathrm{C}(29)$ & $-178.6(3)$ \\
\hline$C(26)$ & $C(25)$ & $C(30)$ & $\mathrm{C}(29)$ & $2.6(5)$ & $\mathrm{C}(17)$ & $\mathrm{C}(12)$ & $\mathrm{C}(11)$ & $\mathrm{C}(10)$ & $1.6(5)$ \\
\hline $\mathrm{C}(13)$ & $\mathrm{C}(12)$ & $\mathrm{C}(11)$ & $\mathrm{C}(10)$ & $-178.1(3)$ & $\mathrm{C}(17)$ & $\mathrm{C}(12)$ & $\mathrm{C}(13)$ & $\mathrm{C}(14)$ & $-0.8(5)$ \\
\hline $\mathrm{C}(11)$ & $\mathrm{C}(12)$ & $\mathrm{C}(13)$ & $\mathrm{C}(14)$ & $178.9(3)$ & $C(8)$ & $\mathrm{C}(9)$ & $C(10)$ & $\mathrm{C}(11)$ & $-2.5(5)$ \\
\hline $\operatorname{Si}(1)$ & $\mathrm{C}(2)$ & $\mathrm{C}(3)$ & $\mathrm{C}(4)$ & $-178.7(3)$ & $C(7)$ & $\mathrm{C}(2)$ & $C(3)$ & $\mathrm{C}(4)$ & $-0.8(4)$ \\
\hline $\operatorname{Si}(1)$ & $\mathrm{C}(2)$ & $\mathrm{C}(7)$ & $\mathrm{C}(6)$ & 179.7(3) & $C(3)$ & $\mathrm{C}(2)$ & $C(7)$ & $C(6)$ & $1.8(5)$ \\
\hline $\mathrm{C}(17)$ & $C(16)$ & $C(15)$ & $\mathrm{C}(14)$ & $0.3(5)$ & $\mathrm{C}(12)$ & $\mathrm{C}(11)$ & $\mathrm{C}(10)$ & $\mathrm{C}(9)$ & $0.7(5)$ \\
\hline$C(25)$ & $C(26)$ & $C(27)$ & $\mathrm{C}(28)$ & $0.5(5)$ & $C(16)$ & $\mathrm{C}(15)$ & $C(14)$ & $\mathrm{C}(13)$ & $-2.1(6)$ \\
\hline
\end{tabular}




$\begin{array}{llllllllll}\mathrm{C}(25) & \mathrm{C}(30) & \mathrm{C}(29) & \mathrm{C}(28) & -1.7(5) & \mathrm{C}(19) & \mathrm{C}(24) & \mathrm{C}(23) & \mathrm{C}(22) & 0.2(5) \\ \mathrm{C}(12) & \mathrm{C}(13) & \mathrm{C}(14) & \mathrm{C}(15) & 2.3(6) & \mathrm{C}(19) & \mathrm{C}(20) & \mathrm{C}(21) & \mathrm{C}(22) & -1.3(6) \\ \mathrm{C}(2) & \mathrm{C}(3) & \mathrm{C}(4) & \mathrm{C}(5) & -0.7(6) & \mathrm{C}(20) & \mathrm{C}(21) & \mathrm{C}(22) & \mathrm{C}(23) & 1.7(6) \\ \mathrm{C}(2) & \mathrm{C}(7) & \mathrm{C}(6) & \mathrm{C}(5) & -1.3(6) & \mathrm{C}(26) & \mathrm{C}(27) & \mathrm{C}(28) & \mathrm{C}(29) & 0.5(5)\end{array}$


Table 8. Torsion angles $\left({ }^{\circ}\right)$-- continued

$\begin{array}{llllllllll}\text { atom1 } & \text { atom2 } & \text { atom3 } & \text { atom4 } & \text { angle } & \text { atom1 } & \text { atom2 } & \text { atom3 } & \text { atom4 } & \text { angle } \\ \mathrm{C}(27) & \mathrm{C}(28) & \mathrm{C}(29) & \mathrm{C}(30) & 0.1(4) & \mathrm{C}(21) & \mathrm{C}(22) & \mathrm{C}(23) & \mathrm{C}(24) & -1.2(6) \\ \mathrm{C}(3) & \mathrm{C}(4) & \mathrm{C}(5) & \mathrm{C}(6) & 1.2(6) & \mathrm{C}(7) & \mathrm{C}(6) & \mathrm{C}(5) & \mathrm{C}(4) & -0.2(5)\end{array}$

The sign is positive if when looking from atom 2 to atom 3 a clock-wise motion of atom 1 would superimpose it on atom 4. 
Table 9. Torsion Angles involving hydrogens $\left({ }^{\circ}\right)$

\begin{tabular}{|c|c|c|c|c|c|c|c|c|c|}
\hline atom1 & atom2 & atom3 & atom4 & angle & atom1 & atom2 & atom3 & atom4 & angle \\
\hline $\mathrm{Si}(1)$ & $\operatorname{Si}(2)$ & $\mathrm{C}(18)$ & $\mathrm{H}(16)$ & -64.5 & $\mathrm{Si}(1)$ & $\operatorname{Si}(2)$ & $\mathrm{C}(18)$ & $\mathrm{H}(17)$ & 55.5 \\
\hline $\operatorname{Si}(1)$ & $\operatorname{Si}(2)$ & $\mathrm{C}(18)$ & $\mathrm{H}(18)$ & 175.7 & $C(19)$ & $\operatorname{Si}(2)$ & $\mathrm{C}(18)$ & $\mathrm{H}(16)$ & 59.9 \\
\hline$C(19)$ & $\operatorname{Si}(2)$ & $\mathrm{C}(18)$ & $\mathrm{H}(17)$ & 179.9 & $\mathrm{C}(19)$ & $\operatorname{Si}(2)$ & $\mathrm{C}(18)$ & $\mathrm{H}(18)$ & -60.0 \\
\hline$C(25)$ & $\operatorname{Si}(2)$ & $\mathrm{C}(18)$ & $\mathrm{H}(16)$ & 178.7 & $\mathrm{C}(25)$ & $\operatorname{Si}(2)$ & $\mathrm{C}(18)$ & $\mathrm{H}(17)$ & -61.3 \\
\hline$C(25)$ & $\operatorname{Si}(2)$ & $\mathrm{C}(18)$ & $\mathrm{H}(18)$ & 58.8 & $\operatorname{Si}(2)$ & $\mathrm{Si}(1)$ & $\mathrm{C}(1)$ & $\mathrm{H}(1)$ & 68.1 \\
\hline $\mathrm{Si}(2)$ & $\mathrm{Si}(1)$ & $\mathrm{C}(1)$ & $\mathrm{H}(2)$ & -171.7 & $\mathrm{Si}(2)$ & $\mathrm{Si}(1)$ & $\mathrm{C}(1)$ & $\mathrm{H}(3)$ & -52.4 \\
\hline $\mathrm{C}(8)$ & $\operatorname{Si}(1)$ & $\mathrm{C}(1)$ & $\mathrm{H}(1)$ & -61.1 & $\mathrm{C}(8)$ & $\operatorname{Si}(1)$ & $\mathrm{C}(1)$ & $\mathrm{H}(2)$ & 59.1 \\
\hline $\mathrm{C}(8)$ & $\operatorname{Si}(1)$ & $\mathrm{C}(1)$ & $\mathrm{H}(3)$ & 178.4 & $\mathrm{C}(2)$ & $\operatorname{Si}(1)$ & $\mathrm{C}(1)$ & $\mathrm{H}(1)$ & -180.0 \\
\hline$C(2)$ & $\operatorname{Si}(1)$ & $\mathrm{C}(1)$ & $\mathrm{H}(2)$ & -59.7 & $\mathrm{C}(2)$ & $\operatorname{Si}(1)$ & $\mathrm{C}(1)$ & $\mathrm{H}(3)$ & 59.6 \\
\hline $\mathrm{C}(8)$ & $\mathrm{C}(17)$ & $C(16)$ & $\mathrm{H}(15)$ & 4.7 & $\mathrm{C}(12)$ & $\mathrm{C}(17)$ & $C(16)$ & $\mathrm{H}(15)$ & -177.0 \\
\hline $\operatorname{Si}(2)$ & $\mathrm{C}(19)$ & $C(24)$ & $\mathrm{H}(23)$ & 2.9 & $\mathrm{C}(20)$ & $\mathrm{C}(19)$ & $\mathrm{C}(24)$ & $\mathrm{H}(23)$ & -178.0 \\
\hline $\operatorname{Si}(2)$ & C(19) & $\mathrm{C}(20)$ & $\mathrm{H}(19)$ & -0.9 & $\mathrm{C}(24)$ & $\mathrm{C}(19)$ & $\mathrm{C}(20)$ & $\mathrm{H}(19)$ & 179.9 \\
\hline $\operatorname{Si}(1)$ & $\mathrm{C}(8)$ & $\mathrm{C}(9)$ & $\mathrm{H}(9)$ & -1.3 & $\mathrm{C}(17)$ & $\mathrm{C}(8)$ & $\mathrm{C}(9)$ & $\mathrm{H}(9)$ & -179.3 \\
\hline $\operatorname{Si}(2)$ & $\mathrm{C}(25)$ & $C(26)$ & $\mathrm{H}(24)$ & -1.8 & $\mathrm{C}(30)$ & $\mathrm{C}(25)$ & $C(26)$ & $\mathrm{H}(24)$ & 177.0 \\
\hline $\operatorname{Si}(2)$ & $\mathrm{C}(25)$ & $\mathrm{C}(30)$ & $\mathrm{H}(28)$ & 0.1 & $C(26)$ & $\mathrm{C}(25)$ & $\mathrm{C}(30)$ & $\mathrm{H}(28)$ & -178.8 \\
\hline $\mathrm{C}(17)$ & $\mathrm{C}(12)$ & $\mathrm{C}(11)$ & $\mathrm{H}(11)$ & -178.2 & $\mathrm{C}(13)$ & $\mathrm{C}(12)$ & $\mathrm{C}(11)$ & $\mathrm{H}(11)$ & 2.2 \\
\hline$C(17)$ & $C(12)$ & $C(13)$ & $\mathrm{H}(12)$ & 179.0 & $\mathrm{C}(11)$ & $C(12)$ & $\mathrm{C}(13)$ & $\mathrm{H}(12)$ & -1.3 \\
\hline $\mathrm{C}(8)$ & $\mathrm{C}(9)$ & $\mathrm{C}(10)$ & $\mathrm{H}(10)$ & 178.9 & $\mathrm{H}(9)$ & $\mathrm{C}(9)$ & $\mathrm{C}(10)$ & $\mathrm{C}(11)$ & 178.6 \\
\hline $\mathrm{H}(9)$ & $\mathrm{C}(9)$ & $C(10)$ & $\mathrm{H}(10)$ & -0.1 & $\mathrm{Si}(1)$ & $\mathrm{C}(2)$ & $\mathrm{C}(3)$ & $\mathrm{H}(4)$ & 2.2 \\
\hline$C(7)$ & $\mathrm{C}(2)$ & $\mathrm{C}(3)$ & $\mathrm{H}(4)$ & -179.9 & $\mathrm{Si}(1)$ & $\mathrm{C}(2)$ & $\mathrm{C}(7)$ & $\mathrm{H}(8)$ & 1.6 \\
\hline $\mathrm{C}(3)$ & $\mathrm{C}(2)$ & $C(7)$ & $\mathrm{H}(8)$ & -176.3 & $\mathrm{C}(17)$ & $C(16)$ & $\mathrm{C}(15)$ & $\mathrm{H}(14)$ & 177.2 \\
\hline $\mathrm{H}(15)$ & $C(16)$ & $C(15)$ & $C(14)$ & 178.5 & $\mathrm{H}(15)$ & $C(16)$ & $\mathrm{C}(15)$ & $\mathrm{H}(14)$ & -4.6 \\
\hline$C(12)$ & $\mathrm{C}(11)$ & $C(10)$ & $\mathrm{H}(10)$ & 179.4 & $\mathrm{H}(11)$ & $\mathrm{C}(11)$ & $C(10)$ & $\mathrm{C}(9)$ & -179.5 \\
\hline $\mathrm{H}(11)$ & $\mathrm{C}(11)$ & $C(10)$ & $\mathrm{H}(10)$ & -0.8 & $\mathrm{C}(25)$ & $C(26)$ & $\mathrm{C}(27)$ & $\mathrm{H}(25)$ & -179.3 \\
\hline $\mathrm{H}(24)$ & $C(26)$ & $C(27)$ & $C(28)$ & -178.5 & $\mathrm{H}(24)$ & $C(26)$ & $\mathrm{C}(27)$ & $\mathrm{H}(25)$ & 1.6 \\
\hline$C(16)$ & $C(15)$ & $C(14)$ & $\mathrm{H}(13)$ & 178.8 & $\mathrm{H}(14)$ & $C(15)$ & $\mathrm{C}(14)$ & $C(13)$ & -179.0 \\
\hline $\mathrm{H}(14)$ & $\mathrm{C}(15)$ & $\mathrm{C}(14)$ & $\mathrm{H}(13)$ & 2.0 & $\mathrm{C}(25)$ & $\mathrm{C}(30)$ & C(29) & $\mathrm{H}(27)$ & 179.7 \\
\hline $\mathrm{H}(28)$ & $\mathrm{C}(30)$ & C(29) & $C(28)$ & 179.7 & $\mathrm{H}(28)$ & $\mathrm{C}(30)$ & $\mathrm{C}(29)$ & $\mathrm{H}(27)$ & 1.1 \\
\hline $\mathrm{C}(19)$ & $\mathrm{C}(24)$ & $\mathrm{C}(23)$ & $\mathrm{H}(22)$ & 176.4 & $\mathrm{H}(23)$ & $\mathrm{C}(24)$ & $\mathrm{C}(23)$ & $\mathrm{C}(22)$ & 178.4 \\
\hline $\mathrm{H}(23)$ & $\mathrm{C}(24)$ & $\mathrm{C}(23)$ & $\mathrm{H}(22)$ & -5.5 & $\mathrm{C}(12)$ & $\mathrm{C}(13)$ & $\mathrm{C}(14)$ & $\mathrm{H}(13)$ & -178.6 \\
\hline $\mathrm{H}(12)$ & $C(13)$ & $C(14)$ & $C(15)$ & -177.5 & $\mathrm{H}(12)$ & $C(13)$ & $C(14)$ & $\mathrm{H}(13)$ & 1.6 \\
\hline C(19) & $C(20)$ & $\mathrm{C}(21)$ & $\mathrm{H}(20)$ & 179.8 & $\mathrm{H}(19)$ & $C(20)$ & $\mathrm{C}(21)$ & $C(22)$ & 179.1 \\
\hline $\mathrm{H}(19)$ & $C(20)$ & $C(21)$ & $\mathrm{H}(20)$ & 0.3 & $\mathrm{C}(2)$ & $\mathrm{C}(3)$ & $\mathrm{C}(4)$ & $\mathrm{H}(5)$ & 178.2 \\
\hline
\end{tabular}




$\begin{array}{llllllllll}\mathrm{H}(4) & \mathrm{C}(3) & \mathrm{C}(4) & \mathrm{C}(5) & 178.3 & \mathrm{H}(4) & \mathrm{C}(3) & \mathrm{C}(4) & \mathrm{H}(5) & -2.8 \\ \mathrm{C}(20) & \mathrm{C}(21) & \mathrm{C}(22) & \mathrm{H}(21) & 178.6 & \mathrm{H}(20) & \mathrm{C}(21) & \mathrm{C}(22) & \mathrm{C}(23) & -179.5 \\ \mathrm{H}(20) & \mathrm{C}(21) & \mathrm{C}(22) & \mathrm{H}(21) & -2.6 & \mathrm{C}(2) & \mathrm{C}(7) & \mathrm{C}(6) & \mathrm{H}(7) & 177.6 \\ \mathrm{H}(8) & \mathrm{C}(7) & \mathrm{C}(6) & \mathrm{C}(5) & 176.7 & \mathrm{H}(8) & \mathrm{C}(7) & \mathrm{C}(6) & \mathrm{H}(7) & -4.3\end{array}$


Table 9. Torsion angles involving hydrogens $\left(^{\circ}\right)$-- continued

$\begin{array}{llllllllll}\text { atom1 } & \text { atom2 } & \text { atom3 } & \text { atom4 } & \text { angle } & \text { atom1 } & \text { atom2 } & \text { atom3 } & \text { atom4 } & \text { angle } \\ \mathrm{C}(26) & \mathrm{C}(27) & \mathrm{C}(28) & \mathrm{H}(26) & -178.7 & \mathrm{H}(25) & \mathrm{C}(27) & \mathrm{C}(28) & \mathrm{C}(29) & -179.7 \\ \mathrm{H}(25) & \mathrm{C}(27) & \mathrm{C}(28) & \mathrm{H}(26) & 1.2 & \mathrm{C}(27) & \mathrm{C}(28) & \mathrm{C}(29) & \mathrm{H}(27) & 178.7 \\ \mathrm{H}(26) & \mathrm{C}(28) & \mathrm{C}(29) & \mathrm{C}(30) & 179.2 & \mathrm{H}(26) & \mathrm{C}(28) & \mathrm{C}(29) & \mathrm{H}(27) & -2.2 \\ \mathrm{C}(21) & \mathrm{C}(22) & \mathrm{C}(23) & \mathrm{H}(22) & -177.3 & \mathrm{H}(21) & \mathrm{C}(22) & \mathrm{C}(23) & \mathrm{C}(24) & -178.1 \\ \mathrm{H}(21) & \mathrm{C}(22) & \mathrm{C}(23) & \mathrm{H}(22) & 5.8 & \mathrm{C}(3) & \mathrm{C}(4) & \mathrm{C}(5) & \mathrm{H}(6) & -178.5 \\ \mathrm{H}(5) & \mathrm{C}(4) & \mathrm{C}(5) & \mathrm{C}(6) & -177.7 & \mathrm{H}(5) & \mathrm{C}(4) & \mathrm{C}(5) & \mathrm{H}(6) & 2.6 \\ \mathrm{C}(7) & \mathrm{C}(6) & \mathrm{C}(5) & \mathrm{H}(6) & 179.5 & \mathrm{H}(7) & \mathrm{C}(6) & \mathrm{C}(5) & \mathrm{C}(4) & -179.1 \\ \mathrm{H}(7) & \mathrm{C}(6) & \mathrm{C}(5) & \mathrm{H}(6) & 0.6 & & & & & \end{array}$

The sign is positive if when looking from atom 2 to atom 3 a clock-wise motion of atom 1 would superimpose it on atom 4. 
Table 10. Distances beyond the asymmetric unit out to $3.60 \AA$

\begin{tabular}{|c|c|c|c|c|c|}
\hline atom & atom & distance & atom & atom & distance \\
\hline $\operatorname{Si}(2)$ & $\mathrm{C}(1)$ & $3.464(3)$ & $\operatorname{Si}(2)$ & $\mathrm{C}(2)$ & $3.334(3)$ \\
\hline $\operatorname{Si}(2)$ & $C(26)$ & $2.857(3)$ & $\mathrm{Si}(2)$ & $C(30)$ & $2.871(3)$ \\
\hline $\operatorname{Si}(2)$ & $\mathrm{C}(24)$ & 2.871(3) & $\mathrm{Si}(2)$ & $C(20)$ & $2.856(3)$ \\
\hline $\operatorname{Si}(1)$ & $\mathrm{C}(17)$ & $2.922(3)$ & $\operatorname{Si}(1)$ & $C(25)$ & $3.454(3)$ \\
\hline $\operatorname{Si}(1)$ & $\mathrm{C}(9)$ & $2.834(3)$ & $\operatorname{Si}(1)$ & $C(16)$ & $3.240(3)$ \\
\hline $\operatorname{Si}(1)$ & $C(18)$ & $3.356(3)$ & $\operatorname{Si}(1)$ & $\mathrm{C}(3)$ & $2.864(3)$ \\
\hline $\operatorname{Si}(1)$ & $\mathrm{C}(7)$ & $2.863(4)$ & $C(17)$ & $\mathrm{C}(9)$ & $2.396(4)$ \\
\hline $\mathrm{C}(17)$ & $\mathrm{C}(11)$ & $2.442(4)$ & $C(17)$ & $C(15)$ & $2.431(5)$ \\
\hline $\mathrm{C}(17)$ & $C(10)$ & $2.788(4)$ & $C(17)$ & $\mathrm{C}(13)$ & $2.455(4)$ \\
\hline $\mathrm{C}(17)$ & $C(14)$ & $2.816(5)$ & $C(19)$ & $C(25)$ & $3.045(4)$ \\
\hline $\mathrm{C}(19)$ & $\mathrm{C}(18)$ & $3.056(4)$ & $C(19)$ & $C(21)$ & $2.423(5)$ \\
\hline $\mathrm{C}(19)$ & $C(22)$ & $2.800(5)$ & $C(19)$ & $C(23)$ & $2.415(4)$ \\
\hline $\mathrm{C}(8)$ & $\mathrm{C}(12)$ & $2.482(4)$ & $\mathrm{C}(8)$ & $\mathrm{C}(1)$ & $3.085(4)$ \\
\hline $\mathrm{C}(8)$ & $\mathrm{C}(2)$ & $3.059(4)$ & $\mathrm{C}(8)$ & $C(16)$ & $2.500(4)$ \\
\hline $\mathrm{C}(8)$ & $\mathrm{C}(11)$ & $2.832(4)$ & $\mathrm{C}(8)$ & $\mathrm{C}(10)$ & $2.439(4)$ \\
\hline $\mathrm{C}(8)$ & $\mathrm{C}(7)$ & $3.585(4)$ & $C(25)$ & $\mathrm{C}(18)$ & $3.088(4)$ \\
\hline $\mathrm{C}(25)$ & $\mathrm{C}(24)$ & $3.289(4)$ & $C(25)$ & $C(27)$ & $2.424(5)$ \\
\hline
\end{tabular}




\begin{tabular}{|c|c|c|c|c|c|}
\hline$C(25)$ & $C(28)$ & $2.799(4)$ & $C(25)$ & $C(29)$ & $2.409(5)$ \\
\hline $\mathrm{C}(12)$ & $\mathrm{C}(9)$ & $2.789(4)$ & $\mathrm{C}(12)$ & $C(16)$ & $2.426(4)$ \\
\hline$C(12)$ & $C(15)$ & $2.782(5)$ & $C(12)$ & $C(10)$ & $2.406(5)$ \\
\hline$C(12)$ & $C(14)$ & $2.403(5)$ & $\mathrm{C}(9)$ & $\mathrm{C}(2)$ & $3.223(4)$ \\
\hline $\mathrm{C}(9)$ & $\mathrm{C}(11)$ & $2.402(5)$ & $\mathrm{C}(9)$ & $\mathrm{C}(7)$ & $3.388(5)$ \\
\hline $\mathrm{C}(1)$ & $\mathrm{C}(2)$ & $3.054(4)$ & $\mathrm{C}(1)$ & $C(16)$ & $3.534(4)$ \\
\hline $\mathrm{C}(1)$ & $\mathrm{C}(3)$ & $3.270(4)$ & $\mathrm{C}(2)$ & $\mathrm{C}(4)$ & $2.425(5)$ \\
\hline $\mathrm{C}(2)$ & $C(6)$ & $2.431(5)$ & $\mathrm{C}(2)$ & $\mathrm{C}(5)$ & $2.805(5)$ \\
\hline$C(16)$ & $C(13)$ & $2.772(5)$ & $C(16)$ & $C(14)$ & $2.397(5)$ \\
\hline $\mathrm{C}(18)$ & $\mathrm{C}(30)$ & $3.359(5)$ & $C(18)$ & $C(20)$ & $3.437(5)$ \\
\hline $\mathrm{C}(11)$ & $\mathrm{C}(13)$ & $2.463(5)$ & $C(26)$ & $C(30)$ & $2.372(4)$ \\
\hline$C(26)$ & $C(24)$ & $3.558(4)$ & $C(26)$ & $C(28)$ & $2.386(5)$ \\
\hline $\mathrm{C}(26)$ & $C(29)$ & $2.741(5)$ & $C(15)$ & $C(13)$ & $2.386(5)$ \\
\hline $\mathrm{C}(30)$ & $C(27)$ & $2.739(5)$ & $C(30)$ & $C(28)$ & $2.382(5)$ \\
\hline$C(24)$ & $C(20)$ & $2.347(5)$ & $C(24)$ & $C(21)$ & $2.730(5)$ \\
\hline$C(24)$ & $C(22)$ & $2.381(5)$ & $C(20)$ & $C(22)$ & $2.382(5)$ \\
\hline $\mathrm{C}(20)$ & $\mathrm{C}(23)$ & $2.728(5)$ & $\mathrm{C}(3)$ & $\mathrm{C}(7)$ & $2.360(5)$ \\
\hline
\end{tabular}




$\begin{array}{llllll}\mathrm{C}(3) & \mathrm{C}(6) & 2.749(5) & \mathrm{C}(3) & \mathrm{C}(5) & 2.393(6) \\ \mathrm{C}(21) & \mathrm{C}(23) & 2.355(6) & \mathrm{C}(7) & \mathrm{C}(4) & 2.730(6) \\ \mathrm{C}(7) & \mathrm{C}(5) & 2.382(6) & & & \\ & & & \mathrm{C}(27) & \mathrm{C}(29) & 2.367(6)\end{array}$


Table 10. Distances beyond the asymmetric unit out to $3.60 \AA$ (continued)

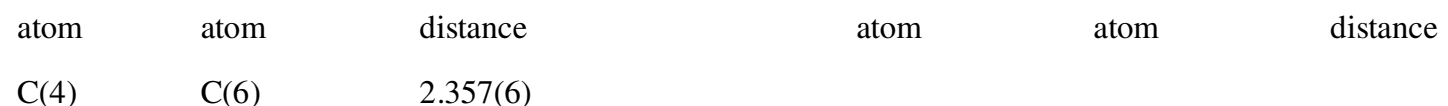

$2.357(6)$ 
Table 11. Distances beyond the asymmetric unit out to $3.60 \AA$ involving hydrogens

\begin{tabular}{|c|c|c|c|c|c|}
\hline atom & atom & distance & atom & atom & distance \\
\hline $\operatorname{Si}(2)$ & $\mathrm{H}(3)$ & 3.4805 & $\mathrm{Si}(2)$ & $\mathrm{H}(8)$ & 3.4820 \\
\hline $\mathrm{Si}(2)$ & $\mathrm{H}(15)$ & 3.2567 & $\mathrm{Si}(2)$ & $\mathrm{H}(16)$ & 2.3647 \\
\hline $\operatorname{Si}(2)$ & $\mathrm{H}(17)$ & 2.3707 & $\mathrm{Si}(2)$ & $\mathrm{H}(18)$ & 2.3662 \\
\hline $\operatorname{Si}(2)$ & $\mathrm{H}(19)$ & 2.9210 & $\mathrm{Si}(2)$ & $\mathrm{H}(23)$ & 2.9445 \\
\hline $\operatorname{Si}(2)$ & $\mathrm{H}(24)$ & 2.9195 & $\mathrm{Si}(2)$ & $\mathrm{H}(28)$ & 2.9443 \\
\hline $\operatorname{Si}(1)$ & $\mathrm{H}(1)$ & 2.3804 & $\operatorname{Si}(1)$ & $\mathrm{H}(2)$ & 2.3565 \\
\hline $\operatorname{Si}(1)$ & $\mathrm{H}(3)$ & 2.3610 & $\operatorname{Si}(1)$ & $\mathrm{H}(4)$ & 2.9326 \\
\hline $\operatorname{Si}(1)$ & $\mathrm{H}(8)$ & 2.9218 & $\mathrm{Si}(1)$ & $\mathrm{H}(9)$ & 2.8767 \\
\hline $\operatorname{Si}(1)$ & $\mathrm{H}(15)$ & 2.7859 & $\operatorname{Si}(1)$ & $\mathrm{H}(16)$ & 3.4555 \\
\hline $\operatorname{Si}(1)$ & $\mathrm{H}(17)$ & 3.3805 & $\operatorname{Si}(1)$ & $\mathrm{H}(24)$ & 3.4054 \\
\hline $\mathrm{C}(17)$ & $\mathrm{H}(1)$ & 3.4471 & $\mathrm{C}(17)$ & $\mathrm{H}(6)^{1)}$ & 3.5704 \\
\hline $\mathrm{C}(17)$ & $\mathrm{H}(9)$ & 3.2550 & $\mathrm{C}(17)$ & $\mathrm{H}(11)$ & 3.3051 \\
\hline $\mathrm{C}(17)$ & $\mathrm{H}(12)$ & 3.3121 & $\mathrm{C}(17)$ & $\mathrm{H}(14)$ & 3.2814 \\
\hline $\mathrm{C}(17)$ & $\mathrm{H}(15)$ & 2.0468 & $\mathrm{C}(17)$ & $\mathrm{H}(24)$ & 3.3210 \\
\hline$C(19)$ & $\mathrm{H}(12)^{2)}$ & 3.3424 & $\mathrm{C}(19)$ & $\mathrm{H}(15)$ & 2.8401 \\
\hline $\mathrm{C}(19)$ & $\mathrm{H}(16)$ & 3.1925 & $\mathrm{C}(19)$ & $\mathrm{H}(18)$ & 3.1949 \\
\hline$C(19)$ & $\mathrm{H}(19)$ & 2.0227 & $\mathrm{C}(19)$ & $\mathrm{H}(20)$ & 3.2743 \\
\hline C(19) & $\mathrm{H}(22)$ & 3.2660 & $\mathrm{C}(19)$ & $\mathrm{H}(23)$ & 2.0122 \\
\hline $\mathrm{C}(19)$ & $\mathrm{H}(24)$ & 3.5699 & $\mathrm{C}(8)$ & $\mathrm{H}(1)$ & 3.2531 \\
\hline $\mathrm{C}(8)$ & $\mathrm{H}(2)$ & 3.2120 & $\mathrm{C}(8)$ & $\mathrm{H}(8)$ & 3.3565 \\
\hline $\mathrm{C}(8)$ & $\mathrm{H}(9)$ & 2.0063 & $\mathrm{C}(8)$ & $\mathrm{H}(10)$ & 3.2833 \\
\hline $\mathrm{C}(8)$ & $\mathrm{H}(15)$ & 2.6618 & $\mathrm{C}(8)$ & $\mathrm{H}(24)$ & 3.0338 \\
\hline$C(25)$ & $\mathrm{H}(8)$ & 2.9599 & $\mathrm{C}(25)$ & $\mathrm{H}(10)^{1)}$ & 3.5131 \\
\hline$C(25)$ & $\mathrm{H}(17)$ & 3.2501 & $\mathrm{C}(25)$ & $\mathrm{H}(18)$ & 3.2269 \\
\hline$C(25)$ & $\mathrm{H}(23)$ & 2.8049 & $\mathrm{C}(25)$ & $\mathrm{H}(24)$ & 2.0374 \\
\hline$C(25)$ & $\mathrm{H}(25)$ & 3.2780 & $\mathrm{C}(25)$ & $\mathrm{H}(27)$ & 3.2630 \\
\hline$C(25)$ & $\mathrm{H}(28)$ & 2.0157 & $\mathrm{C}(12)$ & $\mathrm{H}(6)^{1)}$ & 3.0087 \\
\hline $\mathrm{C}(12)$ & $\mathrm{H}(10)$ & 3.2665 & $\mathrm{C}(12)$ & $\mathrm{H}(11)$ & 2.0619 \\
\hline $\mathrm{C}(12)$ & $\mathrm{H}(12)$ & 2.0540 & $\mathrm{C}(12)$ & $\mathrm{H}(13)$ & 3.2621 \\
\hline $\mathrm{C}(12)$ & $\mathrm{H}(15)$ & 3.2805 & $\mathrm{C}(9)$ & $\mathrm{H}(8)$ & 3.1723 \\
\hline $\mathrm{C}(9)$ & $\mathrm{H}(10)$ & 2.0530 & $\mathrm{C}(9)$ & $\mathrm{H}(11)$ & 3.2571 \\
\hline $\mathrm{C}(9)$ & $\mathrm{H}(23)^{3)}$ & 3.2085 & $\mathrm{C}(9)$ & $\mathrm{H}(24)$ & 3.5313 \\
\hline $\mathrm{C}(1)$ & $\mathrm{H}(4)$ & 2.7552 & $\mathrm{C}(1)$ & $\mathrm{H}(15)$ & 2.9489 \\
\hline
\end{tabular}




$\begin{array}{llllll}\mathrm{C}(1) & \mathrm{H}(16) & 3.5101 & \mathrm{C}(1) & \mathrm{H}(19) & 3.5991 \\ \mathrm{C}(1) & \mathrm{H}(26)^{4)} & 3.4941 & \mathrm{C}(2) & \mathrm{H}(2) & 3.1825 \\ \mathrm{C}(2) & \mathrm{H}(3) & 3.1862 & \mathrm{C}(2) & \mathrm{H}(4) & 2.0234 \\ \mathrm{C}(2) & \mathrm{H}(5) & 3.2788 & \mathrm{C}(2) & \mathrm{H}(7) & 3.2800\end{array}$


Table 11. Distances beyond the asymmetric unit out to $3.60 \AA$ A involving hydrogens -- continued

\begin{tabular}{|c|c|c|c|c|c|}
\hline atom & atom & distance & atom & atom & distance \\
\hline$C(2)$ & $\mathrm{H}(8)$ & 2.0143 & $\mathrm{C}(2)$ & $\mathrm{H}(9)$ & 2.6757 \\
\hline $\mathrm{C}(2)$ & $\mathrm{H}(17)$ & 3.1646 & $\mathrm{C}(2)$ & $\mathrm{H}(25)^{3)}$ & 3.5279 \\
\hline$C(16)$ & $\mathrm{H}(1)$ & 2.9904 & $C(16)$ & $\mathrm{H}(7)^{1)}$ & 3.4804 \\
\hline$C(16)$ & $\mathrm{H}(13)$ & 3.2481 & $C(16)$ & $\mathrm{H}(14)$ & 2.0055 \\
\hline$C(16)$ & $\mathrm{H}(26)^{4)}$ & 3.2373 & $\mathrm{C}(18)$ & $\mathrm{H}(3)$ & 3.4399 \\
\hline $\mathrm{C}(18)$ & $\mathrm{H}(13)^{2)}$ & 3.3907 & $\mathrm{C}(18)$ & $\mathrm{H}(19)$ & 3.0950 \\
\hline $\mathrm{C}(18)$ & $\mathrm{H}(28)$ & 2.8729 & $\mathrm{C}(11)$ & $H(5)^{5)}$ & 3.0731 \\
\hline $\mathrm{C}(11)$ & $H(6)^{1)}$ & 3.2453 & $\mathrm{C}(11)$ & $\mathrm{H}(9)$ & 3.2498 \\
\hline $\mathrm{C}(11)$ & $\mathrm{H}(10)$ & 2.0092 & $\mathrm{C}(11)$ & $\mathrm{H}(12)$ & 2.6461 \\
\hline$C(26)$ & $\mathrm{H}(8)$ & 3.0380 & $C(26)$ & $\mathrm{H}(9)^{1)}$ & 3.3405 \\
\hline$C(26)$ & $\mathrm{H}(22)^{3)}$ & 3.4568 & $C(26)$ & $\mathrm{H}(23)$ & 2.8256 \\
\hline$C(26)$ & $\mathrm{H}(25)$ & 2.0269 & $C(26)$ & $\mathrm{H}(26)$ & 3.2432 \\
\hline$C(26)$ & $\mathrm{H}(28)$ & 3.2309 & $C(15)$ & $\mathrm{H}(7)^{1)}$ & 3.2711 \\
\hline$C(15)$ & $\mathrm{H}(12)$ & 3.2425 & $\mathrm{C}(15)$ & $\mathrm{H}(13)$ & 2.0414 \\
\hline$C(15)$ & $\mathrm{H}(15)$ & 2.0189 & $\mathrm{C}(15)$ & $H(26)^{4)}$ & 3.3731 \\
\hline$C(30)$ & $\mathrm{H}(8)$ & 3.4774 & $\mathrm{C}(30)$ & $\mathrm{H}(10)^{1)}$ & 3.1859 \\
\hline $\mathrm{C}(30)$ & $\mathrm{H}(13)^{2)}$ & 3.4217 & $\mathrm{C}(30)$ & $\mathrm{H}(17)$ & 3.3555 \\
\hline $\mathrm{C}(30)$ & $\mathrm{H}(18)$ & 3.2002 & $\mathrm{C}(30)$ & $\mathrm{H}(24)$ & 3.2301 \\
\hline$C(30)$ & $\mathrm{H}(26)$ & 3.2420 & $\mathrm{C}(30)$ & $\mathrm{H}(27)$ & 2.0242 \\
\hline $\mathrm{C}(24)$ & $\mathrm{H}(7)^{1)}$ & 3.5264 & $\mathrm{C}(24)$ & $H(9)^{1)}$ & 3.3385 \\
\hline$C(24)$ & $\mathrm{H}(10)^{1)}$ & 3.3973 & $\mathrm{C}(24)$ & $\mathrm{H}(12)^{2)}$ & 3.2419 \\
\hline$C(24)$ & $\mathrm{H}(15)$ & 3.3429 & $\mathrm{C}(24)$ & $\mathrm{H}(19)$ & 3.2087 \\
\hline$C(24)$ & $\mathrm{H}(21)$ & 3.2436 & $\mathrm{C}(24)$ & $\mathrm{H}(22)$ & 2.0341 \\
\hline $\mathrm{C}(24)$ & $\mathrm{H}(24)$ & 3.4285 & $\mathrm{C}(10)$ & $H(5)^{5)}$ & 3.4118 \\
\hline$C(10)$ & $\mathrm{H}(9)$ & 2.0506 & $\mathrm{C}(10)$ & $\mathrm{H}(11)$ & 2.0010 \\
\hline$C(10)$ & $H(12)^{6}$ & 3.4580 & $\mathrm{C}(10)$ & $H(13)^{6}$ & 3.5631 \\
\hline$C(10)$ & $\mathrm{H}(23)^{3)}$ & 3.4551 & $\mathrm{C}(13)$ & $\mathrm{H}(6)^{1)}$ & 3.1617 \\
\hline$C(13)$ & $\mathrm{H}(10)^{7)}$ & 3.2878 & $\mathrm{C}(13)$ & $\mathrm{H}(11)$ & 2.6550 \\
\hline$C(13)$ & $\mathrm{H}(13)$ & 2.0051 & $\mathrm{C}(13)$ & $\mathrm{H}(14)$ & 3.2421 \\
\hline$C(20)$ & $\mathrm{H}(12)^{2)}$ & 3.4607 & $\mathrm{C}(20)$ & $\mathrm{H}(15)$ & 3.1048 \\
\hline $\mathrm{C}(20)$ & $\mathrm{H}(16)$ & 3.1879 & $\mathrm{C}(20)$ & $\mathrm{H}(18)$ & 3.5739 \\
\hline $\mathrm{C}(20)$ & $\mathrm{H}(20)$ & 2.0357 & $\mathrm{C}(20)$ & $\mathrm{H}(21)$ & 3.2406 \\
\hline$C(20)$ & $\mathrm{H}(23)$ & 3.2080 & $\mathrm{C}(20)$ & $H(26)^{4)}$ & 3.1944 \\
\hline
\end{tabular}




$\begin{array}{llllll}\mathrm{C}(20) & \mathrm{H}(27)^{4)} & 3.4031 & \mathrm{C}(3) & \mathrm{H}(2) & 3.1659 \\ \mathrm{C}(3) & \mathrm{H}(3) & 3.1545 & \mathrm{C}(3) & \mathrm{H}(5) & 2.0451 \\ \mathrm{C}(3) & \mathrm{H}(6) & 3.2566 & \mathrm{C}(3) & \mathrm{H}(8) & 3.2154 \\ \mathrm{C}(3) & \mathrm{H}(17) & 3.5375 & \mathrm{C}(3) & \mathrm{H}(25)^{3)} & 3.0033\end{array}$


Table 11. Distances beyond the asymmetric unit out to $3.60 \AA$ Anvolving hydrogens -- continued

\begin{tabular}{|c|c|c|c|c|c|}
\hline atom & atom & distance & atom & atom & distance \\
\hline$C(14)$ & $\mathrm{H}(7)^{1)}$ & 3.3637 & $\mathrm{C}(14)$ & $\mathrm{H}(10)^{7)}$ & 3.2288 \\
\hline$C(14)$ & $\mathrm{H}(12)$ & 1.9997 & $\mathrm{C}(14)$ & $\mathrm{H}(14)$ & 2.0490 \\
\hline $\mathrm{C}(14)$ & $\mathrm{H}(15)$ & 3.2579 & $\mathrm{C}(14)$ & $\mathrm{H}(18)^{8)}$ & 3.0320 \\
\hline$C(21)$ & $\mathrm{H}(12)^{2)}$ & 3.5322 & $\mathrm{C}(21)$ & $\mathrm{H}(19)$ & 2.0294 \\
\hline $\mathrm{C}(21)$ & $\mathrm{H}(21)$ & 2.0119 & $\mathrm{C}(21)$ & $\mathrm{H}(22)$ & 3.2108 \\
\hline $\mathrm{C}(21)$ & $H(26)^{4)}$ & 3.3186 & $\mathrm{C}(21)$ & $\mathrm{H}(27)^{4)}$ & 3.5868 \\
\hline$C(7)$ & $\mathrm{H}(4)$ & 3.2195 & $C(7)$ & $\mathrm{H}(6)$ & 3.2385 \\
\hline$C(7)$ & $\mathrm{H}(7)$ & 2.0386 & $C(7)$ & $\mathrm{H}(9)$ & 2.6793 \\
\hline$C(7)$ & $\mathrm{H}(17)$ & 3.5674 & $\mathrm{C}(7)$ & $\mathrm{H}(22)^{3)}$ & 3.5430 \\
\hline $\mathrm{C}(7)$ & $\mathrm{H}(23)^{3)}$ & 3.2353 & $\mathrm{C}(27)$ & $\mathrm{H}(9)^{1)}$ & 3.2113 \\
\hline$C(27)$ & $\mathrm{H}(22)^{3)}$ & 3.0792 & $C(27)$ & $\mathrm{H}(24)$ & 2.0311 \\
\hline$C(27)$ & $\mathrm{H}(26)$ & 2.0194 & $C(27)$ & $\mathrm{H}(27)$ & 3.2223 \\
\hline $\mathrm{C}(28)$ & $\mathrm{H}(1)^{9)}$ & 3.0505 & $\mathrm{C}(28)$ & $\mathrm{H}(9)^{1)}$ & 3.4479 \\
\hline $\mathrm{C}(28)$ & $\mathrm{H}(19)^{9)}$ & 3.5743 & $\mathrm{C}(28)$ & $\mathrm{H}(20)^{9)}$ & 3.5943 \\
\hline $\mathrm{C}(28)$ & $\mathrm{H}(24)$ & 3.2423 & $\mathrm{C}(28)$ & $\mathrm{H}(25)$ & 2.0183 \\
\hline $\mathrm{C}(28)$ & $\mathrm{H}(27)$ & 2.0160 & $\mathrm{C}(28)$ & $\mathrm{H}(28)$ & 3.2370 \\
\hline$C(22)$ & $\mathrm{H}(11)^{2)}$ & 3.4997 & $C(22)$ & $\mathrm{H}(12)^{2)}$ & 3.4456 \\
\hline$C(22)$ & $\mathrm{H}(19)$ & 3.2335 & $\mathrm{C}(22)$ & $\mathrm{H}(20)$ & 2.0137 \\
\hline$C(22)$ & $\mathrm{H}(22)$ & 2.0094 & $\mathrm{C}(22)$ & $\mathrm{H}(23)$ & 3.2329 \\
\hline $\mathrm{C}(4)$ & $\mathrm{H}(4)$ & 2.0465 & $\mathrm{C}(4)$ & $\mathrm{H}(6)$ & 2.0187 \\
\hline$C(4)$ & $\mathrm{H}(7)$ & 3.2179 & $\mathrm{C}(4)$ & $\mathrm{H}(11)^{10)}$ & 3.5869 \\
\hline $\mathrm{C}(4)$ & $H(25)^{3)}$ & 2.8961 & $C(6)$ & $H(5)$ & 3.2116 \\
\hline$C(6)$ & $\mathrm{H}(6)$ & 2.0076 & $C(6)$ & $\mathrm{H}(8)$ & 2.0373 \\
\hline$C(6)$ & $\mathrm{H}(9)$ & 3.6000 & $\mathrm{C}(6)$ & $\mathrm{H}(23)^{3)}$ & 3.3037 \\
\hline$C(6)$ & $\mathrm{H}(24)^{3)}$ & 3.3169 & $C(29)$ & $\mathrm{H}(1)^{9)}$ & 3.5092 \\
\hline $\mathrm{C}(29)$ & $\mathrm{H}(10)^{1)}$ & 3.4196 & $\mathrm{C}(29)$ & $H(19)^{9)}$ & 3.4465 \\
\hline$C(29)$ & $\mathrm{H}(20)^{9)}$ & 3.5220 & $C(29)$ & $\mathrm{H}(25)$ & 3.2267 \\
\hline$C(29)$ & $\mathrm{H}(26)$ & 2.0286 & $\mathrm{C}(29)$ & $\mathrm{H}(28)$ & 2.0193 \\
\hline $\mathrm{C}(23)$ & $\mathrm{H}(8)^{1)}$ & 3.4576 & $\mathrm{C}(23)$ & $\mathrm{H}(12)^{2)}$ & 3.3060 \\
\hline$C(23)$ & $\mathrm{H}(20)$ & 3.2147 & $\mathrm{C}(23)$ & $\mathrm{H}(21)$ & 2.0208 \\
\hline $\mathrm{C}(23)$ & $\mathrm{H}(23)$ & 2.0255 & $C(5)$ & $\mathrm{H}(4)$ & 3.2478 \\
\hline$C(5)$ & $\mathrm{H}(5)$ & 2.0038 & $C(5)$ & $\mathrm{H}(7)$ & 2.0191 \\
\hline $\mathrm{C}(5)$ & $\mathrm{H}(8)$ & 3.2370 & $\mathrm{C}(5)$ & $\mathrm{H}(24)^{3)}$ & 3.2694 \\
\hline
\end{tabular}




$\begin{array}{lll}\mathrm{C}(5) & \mathrm{H}(25)^{3)} & 3.3126 \\ \mathrm{H}(1) & \mathrm{C}(29)^{4)} & 3.5092 \\ \mathrm{H}(5) & \mathrm{C}(10)^{10)} & 3.4118 \\ \mathrm{H}(6) & \mathrm{C}(12)^{3)} & 3.0087\end{array}$

$\mathrm{H}(1)$

$\mathrm{C}(28)^{4)}$

3.0505

H(5)

$\mathrm{C}(11)^{10)}$

3.0731

H(6)

$\mathrm{C}(17)^{3)}$

3.5704

H(6)

$\mathrm{C}(11)^{3)}$

3.2453 
Table 11. Distances beyond the asymmetric unit out to $3.60 \AA$ A involving hydrogens -- continued

\begin{tabular}{|c|c|c|c|c|c|}
\hline atom & atom & distance & atom & atom & distance \\
\hline $\mathrm{H}(6)$ & $\mathrm{C}(13)^{3)}$ & 3.1617 & $\mathrm{H}(7)$ & $C(16)^{3)}$ & 3.4804 \\
\hline $\mathrm{H}(7)$ & $C(15)^{3)}$ & 3.2711 & $\mathrm{H}(7)$ & $\mathrm{C}(24)^{3)}$ & 3.5264 \\
\hline $\mathrm{H}(7)$ & $\mathrm{C}(14)^{3)}$ & 3.3637 & $\mathrm{H}(8)$ & $\mathrm{C}(23)^{3)}$ & 3.4576 \\
\hline $\mathrm{H}(9)$ & $C(26)^{3)}$ & 3.3405 & $\mathrm{H}(9)$ & $\mathrm{C}(24)^{3)}$ & 3.3385 \\
\hline $\mathrm{H}(9)$ & $\mathrm{C}(27)^{3)}$ & 3.2113 & $\mathrm{H}(9)$ & $\mathrm{C}(28)^{3)}$ & 3.4479 \\
\hline $\mathrm{H}(10)$ & $C(25)^{3)}$ & 3.5131 & $\mathrm{H}(10)$ & $\mathrm{C}(30)^{3)}$ & 3.1859 \\
\hline $\mathrm{H}(10)$ & $C(24)^{3)}$ & 3.3973 & $\mathrm{H}(10)$ & $\mathrm{C}(13)^{6}$ & 3.2878 \\
\hline $\mathrm{H}(10)$ & $C(14)^{6)}$ & 3.2288 & $\mathrm{H}(10)$ & $\mathrm{C}(29)^{3)}$ & 3.4196 \\
\hline $\mathrm{H}(11)$ & $C(22)^{8)}$ & 3.4997 & $\mathrm{H}(11)$ & $\mathrm{C}(4)^{5)}$ & 3.5869 \\
\hline $\mathrm{H}(12)$ & $C(19)^{8)}$ & 3.3424 & $\mathrm{H}(12)$ & $C(24)^{8)}$ & 3.2419 \\
\hline $\mathrm{H}(12)$ & $C(10)^{7)}$ & 3.4580 & $\mathrm{H}(12)$ & $\mathrm{C}(20)^{8)}$ & 3.4607 \\
\hline $\mathrm{H}(12)$ & $C(21)^{8)}$ & 3.5322 & $\mathrm{H}(12)$ & $C(22)^{8)}$ & 3.4456 \\
\hline $\mathrm{H}(12)$ & $C(23)^{8)}$ & 3.3060 & $\mathrm{H}(13)$ & $\mathrm{C}(18)^{8)}$ & 3.3907 \\
\hline $\mathrm{H}(13)$ & $C(30)^{8)}$ & 3.4217 & $\mathrm{H}(13)$ & $C(10)^{7)}$ & 3.5631 \\
\hline $\mathrm{H}(18)$ & $\mathrm{C}(14)^{2)}$ & 3.0320 & $\mathrm{H}(19)$ & $C(28)^{4)}$ & 3.5743 \\
\hline $\mathrm{H}(19)$ & $C(29)^{4)}$ & 3.4465 & $\mathrm{H}(20)$ & $C(28)^{4)}$ & 3.5943 \\
\hline $\mathrm{H}(20)$ & $C(29)^{4)}$ & 3.5220 & $\mathrm{H}(22)$ & $C(26)^{1)}$ & 3.4568 \\
\hline $\mathrm{H}(22)$ & $\mathrm{C}(7)^{1)}$ & 3.5430 & $\mathrm{H}(22)$ & $\mathrm{C}(27)^{1)}$ & 3.0792 \\
\hline $\mathrm{H}(23)$ & $\mathrm{C}(9)^{1)}$ & 3.2085 & $\mathrm{H}(23)$ & $C(10)^{1)}$ & 3.4551 \\
\hline $\mathrm{H}(23)$ & $\mathrm{C}(7)^{1)}$ & 3.2353 & $\mathrm{H}(23)$ & $C(6)^{1)}$ & 3.3037 \\
\hline $\mathrm{H}(24)$ & $C(6)^{1)}$ & 3.3169 & $\mathrm{H}(24)$ & $C(5)^{1)}$ & 3.2694 \\
\hline $\mathrm{H}(25)$ & $\mathrm{C}(2)^{1)}$ & 3.5279 & $\mathrm{H}(25)$ & $\mathrm{C}(3)^{1)}$ & 3.0033 \\
\hline $\mathrm{H}(25)$ & $\mathrm{C}(4)^{1)}$ & 2.8961 & $\mathrm{H}(25)$ & $C(5)^{1)}$ & 3.3126 \\
\hline $\mathrm{H}(26)$ & $C(1)^{9)}$ & 3.4941 & $\mathrm{H}(26)$ & $C(16)^{9)}$ & 3.2373 \\
\hline $\mathrm{H}(26)$ & $C(15)^{9)}$ & 3.3731 & $\mathrm{H}(26)$ & $\mathrm{C}(20)^{9)}$ & 3.1944 \\
\hline $\mathrm{H}(26)$ & $\mathrm{C}(21)^{9)}$ & 3.3186 & $\mathrm{H}(27)$ & $\mathrm{C}(20)^{9)}$ & 3.4031 \\
\hline $\mathrm{H}(27)$ & $\mathrm{C}(21)^{9)}$ & 3.5868 & & & \\
\hline
\end{tabular}

Symmetry Operators:
(1) $-\mathrm{X}+1, \mathrm{Y}+1 / 2-1,-\mathrm{Z}+1 / 2+1$
(2) $-X+1 / 2+1,-Y, Z+1 / 2$
(3) $-\mathrm{X}+1, \mathrm{Y}+1 / 2,-\mathrm{Z}+1 / 2+1$
(4) $\mathrm{X}+1, \mathrm{Y}, \mathrm{Z}$
(5) $-\mathrm{X}+1 / 2+1,-\mathrm{Y}+1, \mathrm{Z}+1 / 2-1$
(6) $\mathrm{X}+1 / 2-1,-\mathrm{Y}+1 / 2,-\mathrm{Z}+1$ 
(7) $\mathrm{X}+1 / 2,-\mathrm{Y}+1 / 2,-\mathrm{Z}+1$

(8) $-\mathrm{X}+1 / 2+1,-\mathrm{Y}, \mathrm{Z}+1 / 2-1$

(9) $\mathrm{X}-1, \mathrm{Y}, \mathrm{Z}$

(10) $-\mathrm{X}+1 / 2+1,-\mathrm{Y}+1, \mathrm{Z}+1 / 2$ 